bioRxiv preprint doi: https://doi.org/10.1101/2021.05.31.446489; this version posted June 1,2021. The copyright holder for this preprint (which was not certified by peer review) is the author/funder, who has granted bioRxiv a license to display the preprint in perpetuity. It is made available under aCC-BY-NC-ND 4.0 International license.

\title{
RNA surveillance controls 3D genome structure via stable cohesin-chromosome interaction
}

2

$$
\text { Yujin Chun }{ }^{1, *} \text {, Sungwook Han }{ }^{1, *} \text {, Taemook Kim }{ }^{1, *} \text {, Yoonjung Choi }{ }^{2, \#} \text { and Daeyoup Lee }{ }^{1, \#}
$$

${ }^{1}$ Department of Biological Sciences, Korea Advanced Institute of Science and Technology, Daejeon 34141, South Korea; ${ }^{2}$ Deargen Inc., Munji-ro, Yuseong-gu, Daejeon, 34051, South Korea

\section{*These authors contributed equally to this work}

\section{\#Corresponding author: Yoonjung Choi and Daeyoup Lee}

${ }^{1}$ Department of Biological Sciences, Korea Advanced Institute of Science and Technology, Daejeon 34141, South Korea. Tel: +82-42-350-2623; Email: daeyoup@kaist.ac.kr

${ }^{2}$ Deargen Inc., Munji-ro, Yuseong-gu, Daejeon, 34051, South Korea. Tel: +82-42-716-2100; Email: yoonjungc@deargen.me

Keywords: Cohesin binding, Transcription termination, nuclear exosome, 3'-end RNA processing, RNA surveillance, 3D genome organization 
The 3D architecture that the genome is folded into is regulated by CTCF, which determines domain borders, and cohesin, which generates interactions within domains. However, organisms lacking CTCF have been reported to still have cohesin-mediated 3D structures with strong borders. How this can be achieved and precisely regulated are yet unknown. Using in situ Hi-C, we found that 3'-end RNA processing factors coupled with proper transcription termination are a cis-acting determinant that regulates the localization and dynamics of cohesin on the chromosome arms. Loss of RNA processing factors, including nuclear exosome and Pfs2, destabilizes cohesin from the 3'-ends of convergent genes and results in decreased cohesin-mediated domain boundaries. We observed the co-localization between Rad21 and a wide range of 3'end RNA processing/termination factors. Further, deletion of Rrp6 leads to cohesin redistribution to facultative heterochromatin, resulting in improper domain boundaries. Importantly, we observed that knockdown of Rrp6 $6^{\text {Exosc10 }}$ caused a defect in cohesin binding and loss of local topologically associating domains (TADs) interactions in mouse embryonic stem cells. Based on these findings, we propose a novel function of the RNA surveillance pathway in 3D genome organization via cohesin complex, which provides the molecular basis underlying the dynamics of cohesin function. 


\section{Introduction}

The mammalian genome is spatially organized into complex three-dimensional (3D) architecture encompassing compartments, topologically associating domains (TADs), and chromatin loops that allow for proper gene regulation (Dixon et al., 2012; Lieberman-Aiden et al., 2009; Nora et al., 2012; Rao et al., 2014). The 3D architecture is characterized by the convergent binding of the CCCTC-binding factor (CTCF) at the boundaries of chromatin domains and by the action of cohesin (Nora et al., 2017; Parelho et al., 2008; Rao et al., 2014; Rubio et al., 2008; Schwarzer et al., 2017; Wutz et al., 2017). The recent loop extrusion model suggested that cohesin extrudes loops until blocked by convergently oriented CTCF, which pre-marks the TAD borders (Fudenberg et al., 2016; Goloborodko et al., 2016; Sanborn et al., 2015). Cohesin and CTCF thereby maintain stable anchoring of chromatin loops and form discrete interaction domains.

Interestingly, these TAD-like structures and chromatin loops are also found in organisms that lack CTCF homologs. From S.cerevisiae to S.pombe, C.elegans, and A. thaliana, these organisms reveal discrete self-interacting domains with varying domain lengths independent of CTCF (Costantino et al., 2020; Hsieh et al., 2015; Rowley et al., 2017; Rowley and Corces, 2016). The cohesin, a ring-shaped protein complex, mediates the stable cohesion of sister chromatids and loop extrusion by holding distant DNA fragments in close spatial proximity (Murayama et al., 2018; Onn et al., 2008). In fission yeast, cohesin peaks are preferentially enriched at 3 '-ends of convergent genes, and cohesin mediates the formation of interaction domains, termed 'globules', to organize chromatin architecture during interphase (Mizuguchi et al., 2014; Tanizawa et al., 2017). Even in Drosophila where CTCF homolog exists, Drosophila genome lacks evidence for the the colocalization of CTCF and cohesin complex as well as CTCF-mediated loop extrusion mechanism (Bartkuhn et al., 2009; Matthews and White, 2019). These findings imply that the formation and maintenance of chromatin interaction domains would require CTCF-independent regulatory factors (e.g., cohesin) or relevant biological processes, such as transcription/chromatin states, RNAPII or other transcription complex.

However, it is not fully resolved how cohesin determines the 3D chromatin organization and what mediates the stable cohesin localization in eukaryotes that lack CTCF or of which the CTCF does not play a major role. The cohesin accumulation at the 3'-end of convergent genes is achieved by passive translocation of cohesin along with RNAPII (Gullerova and Proudfoot, 2008; Schmidt et al., 2009). One possibility is the active role of transcription processes (including initiation, elongation, and termination) in regulating 3D chromatin structure and positioning of cohesin (Bhardwaj et al., 2016). Recent study suggested the importance of proper positioning of cohesin and RNAPII via transcription elongation in the formation of gene loops (Rowley et al., 2019). In addition, defects in transcription elongation cause mislocalization of cohesin and disruption of 3D chromatin interactions (Heinz et al., 2018). However, it has been suggested that not only the transcription elongation process which 
translocates cohesin together with elongating RNAPII, but also the 3'-end RNA processing machinery coupled with transcription termination plays a role in controlling genome topology and genome stability. In yeast, transcription terminator and CPF 3'-end processing machinery were reported to mediate gene looping (O'Sullivan et al., 2004; Tan-Wong et al., 2012). Given that loss of cohesin complex across the chromosomes results in missegregation of chromosomes and lagging chromosomes (Hadjur et al., 2009; Haering et al., 2008; Losada et al., 1998; Peters et al., 2008), involvement of various 3'-end processing and termination related factors in chromosome missegregation (Graham et al., 2009; Kinoshita et al., 1991; Mukarami et al., 2007; Ohkura et al., 1989, 1988; Shobuike et al., 2001; Wang et al., 2005; Win et al., 2006) imply an intimate relationship with cohesin.

Rrp6 and Dis3, which together form the nuclear exosome complex (Liu et al., 2006), are known to be involved in various RNA-related processes, including degradation of irregularly terminated transcripts (Vasiljeva and Buratowski, 2006) and noncoding cryptic unstable transcripts (CUTs) (Colin et al., 2011), and transcription termination of variety of noncoding RNAs (Fox et al., 2015). Along with RNA processing and termination-related functions, they also function in chromosome segregation (Graham et al., 2009; Mukarami et al., 2007). Pfs2, which encodes the $S$. pombe homolog of the WDR33 in mammalian CPSF complex, recognizes AAUAAA polyadenylation site (PAS) together with CPSF30 (Chan et al., 2014; Clerici et al., 2018; Schönemann et al., 2014). Defects in the CPSF complex lead to defects in transcription termination by ultimately causing extensive transcriptional readthrough genome-wide (Eaton et al., 2020). The loss of Pfs2 reportedly results in chromosome missegregation in S. pombe (Wang et al., 2005), indirectly implying a relationship between cohesin and beyond transcription elongation event. Thus, we focused our attention on elucidating the role of 3'-end mRNA processing linked with proper transcription termination at cohesin dynamics and $3 \mathrm{D}$ chromatin structure.

Here, we show that RNA processing factors involved in transcription termination contribute to the CTCF-independent regulation of cohesin localization and dynamics. Importantly, 3'-end RNA processing factors and proper transcription termination enables highly coordinated 3D genome architecture by regulating cohesin-mediated interaction domain formation. We confirmed that this relationship is also conserved in mouse embryonic stem cells. Finally, we provide a new molecular basis for control the dynamics of cohesin biology through uncovering a relationship between mRNA surveillance and cohesin complex. 


\section{Result}

3'-end RNA processing factors regulate the localization of the cohesin complex at the 3'-ends of convergent genes in euchromatin

To explore the relationship between 3 '-end mRNA processing and chromatin localization of cohesin, we performed chromatin immunoprecipitation sequencing (ChIP-seq) for FLAG-tagged $\operatorname{Rad} 21$ (the kleisin subunit of cohesin complex) using mutant yeast with defects in the nuclear exosome complex (Rrp6 and Dis3) and the 3'-end formation of mRNA (Pfs2). Given that Dis3 and Pfs2 are essential and to avoid introducing bias from cell-cycle arrest, we grew the dis3-54 and pfs2-11 mutant cells at their semi-permissive temperatures $\left(30^{\circ} \mathrm{C}\right.$ and $32^{\circ} \mathrm{C}$, respectively). $\operatorname{Rad} 21$ peaks identified in wild type cells showed a dominant $(\sim 45 \%)$ distribution and strong enrichment at the 3 '-ends of convergent genes and are regularly positioned across the genome as previously reported (Schmidt et al., 2009) (Figure 1 - figure supplement 1A). For further analysis, we mainly focused on the Rad21 sites (peaks) at intergenic sites where genes with opposite strands converge (converging intergenic sites). We detected a significant decrease in Rad21 intensity at the 3'-ends of convergent genes in rrp6 and pfs211 (67\% and 58\% fold decrease in rrp6 $\Delta$ and $p f s 2-11$ cells, respectively), compared to those in wt cells (Figure 1A-C). In dis3-54 mutant cells, reduction of Rad21 was relatively weaker than $\operatorname{rrp} 6 \Delta$ due to a weak semi-permissive mutant of Dis3 (Figure 1A-C). Thus, we additionally employed a tetracyclineregulated system (Zilio et al., 2012) to modulate $d i s 3^{+}$expression and check by conventional ChIPqPCR whether the temporal depletion of Dis3 affects Rad21 occupancy. Consistent with previous report (Lemay et al., 2014), depletion of Dis3 by over $~ 80 \%$ caused the severe defect in transcription termination, resulting in readthrough transcripts (Figure 1 - figure supplement 1B-C). Also, we observed the two-fold reduction of Rad21 enrichment upon Dis3 knockdown (Figure 1 - figure supplement 1D). We additionally performed ChIP-seq of FLAG-tagged Psc3 ${ }^{\text {SCC3/STAG1/STAG2 }}$ (an accessory subunit of the cohesin complex) and found that Psc3 occupancy in the rrp6 mutant was significantly reduced (Figure 1 - figure supplement 1E-F). These findings imply that 3'-end RNA processing factors are involved in regulating the occupancy of the cohesin complex at converging intergenic sites.

To further examine our results, we performed Rad21 ChIP-seq in cid14 $\Delta$ and exo $2 \Delta$ mutants. Cid14 is a component of the Cid14/Trf4-Air1-Mtr4 polyadenylation (TRAMP) complex, which facilitates RNA turnover by stimulating exosome activity and contributes to faithful chromosome segregation and establishment of sister chromatid cohesion during the $\mathrm{S}$ phase (Callahan and Butler, 2010; Win et al., 2006). In cid14 $\Delta$ cells, we found a notable decrease in Rad21 levels (Figure 1 - figure

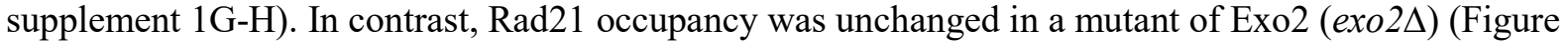
1 - figure supplement 1G-H), which encodes the S. pombe homolog of the XRN1 5'-3' exonuclease (Szankasi and Smith, 1996), indicating that the defect in cytoplasmic 5'-end RNA decay does not affect 
the Rad21 occupancy. While cohesin complex is preferentially enriched at convergent genes and evenly distributed throughout the genome in interphase, pol III-transcribed tRNA is the second most cohesinenriched region and showed distinct cohesin peaks (Figure 1 - figure supplement 1I). Nuclear exosome and TRAMP complex are also well-known to process various RNA substrates, including tRNA, snoRNA, or rRNA. In rrp6 6 or cid14 4 mutants, but not in pfs2-11 mutant cells, Rad21 is indeed disrupted at these tRNA loci, supporting the direct involvement of RNA processing factors in regulating cohesin occupancy at not only in Pol II-dependent genes but also in polI/III-dependent genes in a genome-wide manner.

In S. pombe, Swi6 ${ }^{\mathrm{HP} 1}$ recruits Rad21 to heterochromatin regions by physically interacting with the cohesin complex (Bernard et al., 2001). Consistently, we found by Rad21 ChIP-seq that Rad21 levels at the centromeres and $t h 1^{+}$subtelomeric regions (tellR) decreased in swi6 $\Delta$ cells (Figure 1 figure supplement 2A). However, as reported previously (Bernard et al., 2001; Schmidt et al., 2009), a lack of Swi $6^{\mathrm{HP} 1}$ had no discernible effects on the Rad21 distribution at the chromosome arms (Figure 1 - figure supplement $1 \mathrm{G}-\mathrm{H})$. Thus, this indicates that Rad21 occupancy at the euchromatic chromosome arms is regulated by mechanisms distinct from those at heterochromatin regions.

We then checked whether Rrp6 and related factors were required for cohesin localization at the heterochromatin regions such as the centromere and $t h 1^{+}$subtelomeric regions. Unlike the results obtained from euchromatin, in rrp6 $\Delta$ and $p f_{s} 2-11$ cells, we observed no discernible changes in $\operatorname{Rad} 21$ enrichment at heterochromatin (Figure 1 - figure supplement 2A) except at tRNA locus which resided within the heterochromatin. On the contrary, in cid144 and dis3-54 cells, we found a reduced Rad21 occupancy at heterochromatin, particularly at subtelomeres (Figure 1 - figure supplement 2A), indicating the distinct function of these factors in regulating subtelomere silencing. Moreover, all of the aforementioned changes in Rad21 ChIP-seq intensities were not due to changes in the Rad21 expression (Figure 1 - figure supplement 2B). Altogether, our results emphasize the novel regulatory role of 3'end processing, which enables the stable and proper positioning of cohesin across the chromosome arm, especially at the 3'-end of convergent genes (Figure 1D).

\section{Rrp6 directly regulates cohesin occupancy}

Rrp6 is also known to play a central role in mediating proper termination by interacting with various cofactors (Fox and Mosley, 2016). To evaluate the direct regulation of cohesin localization by 3'-end RNA processing and transcription termination, we focused on Rrp6 to dissect the involvement of Rrp6 in regulating cohesin dynamics and turnover. We temporally depleted Rrp6 and then rescued/restored using a tetracycline-regulated system in a time-course manner (Zilio et al., 2012) (Figure 2A-B). We monitored PK-tagged Rad21 occupancy and discovered that Rad21 enrichment at 
converging intergenic sites (Figure 2C-E) was gradually decreased upon $r r p 6^{+}$knockdown (-ahTet 8 and 12 hours). When we rescued $r r p 6^{+}$expression by adding ahTet (+ahTet 0.5 and 4 hours), $\operatorname{Rad} 21$ occupancy was quickly restored (Figure 2C-E). Thus, the kinetics of $r r p 6^{+}$expression was highly correlated with the binding intensity of Rad21, indicating that the Rrp6-dependent regulation of $\operatorname{Rad} 21$ is reversible and that stabilization of the cohesin complex requires Rrp6.

Wapl, one of the regulatory subunits in the cohesin complex, releases cohesin from chromatin (cohesin is stabilized on the chromatin when Wapl is depleted) and restricts chromatin loop extension (Dauban et al., 2020; Haarhuis et al., 2017; Kueng et al., 2006; Vernì et al., 2000; Wutz et al., 2017). In fission yeast, we examined the interplay between Wapl and Rrp6 in cohesin turnover by combining the deletion mutant of the $w p l l^{+}$with $r r p 6 \Delta$ (Figure 2 - figure supplement 1A). In $w p l l \Delta$ cells, we detected increased cohesin residence at converging intergenic regions, as compared to wt cells (Figure 2F-H). Notably, the reduced binding of Rad21 in rrp6 $\Delta$ cells was partially rescued ( $P$ value $\left.=1.65 \times 10^{-06}\right)$ in the $\operatorname{rrp} 6 \Delta$ wpll $\Delta$ double mutant (Figure $2 \mathrm{G}$ ). These results suggest that Rrp6 is directly involved in governing the turnover of cohesin at converging intergenic sites.

Along with generating contact domains in the G1 phase of the cell cycle (Bernard et al., 2008; Mizuguchi et al., 2014; Tanizawa et al., 2017), cohesin mediates sister chromatid cohesion following the S phase (Haering et al., 2008; Losada et al., 1998; Tóth et al., 1999). Since our results imply a direct linkage between Rrp6 and cohesin occupancy, we speculated that Rrp6 might play a role in the establishment of sister chromatid cohesion along chromosome arms. To prove this, we examined the state of sister chromatid cohesion using strains expressing LacI-GFP bound to LacO repeats inserted at ade $^{+}$sites linked to the chromosome arm (Nabeshima et al., 1998; Yamamoto and Hiraoka, 2003). Wild-type and swi6 $\Delta$ cells usually displayed a single spot of GFP, indicating normal sister chromatid cohesion along the chromosome arms (Figure 2 - figure supplement 1B-C). In contrast, $\operatorname{rrp} 6 \Delta$ cells exhibited a higher proportion of dual GFP foci at the $a d e \sigma^{+}$locus, indicating a defect in arm cohesion (Figure 2 - figure supplement 1B-C). Since Swi6 plays a major role in the centromere-specific accumulation of Rad21, we hypothesized that combining $\operatorname{rrp} 6 \Delta$ and swi $6 \Delta$ would show a synergistic effect. As expected, we observed a synthetic growth defect in the rrp $6 \Delta$ swi $6 \Delta$ double mutant, indicating that the function of Rad21 was synergistically disrupted in these cells (Figure 2 - figure supplement 1D). These data provide an additional clue that Rrp6 is involved in the regulation of sister chromatid cohesion via regulating cohesin occupancy at the chromosome arms.

\section{Nuclear exosome and Pfs2 regulate the higher-order 3D genome architecture via rad21}

To elucidate how the loss of Rad21 occupancy upon defects in 3'-end RNA processing and transcription termination affects the 3D chromatin organization, we performed in situ Hi-C (Rao et al., 
2014) on wt, $\operatorname{rrp} 6 \Delta, p f s 2-11$, and rad21-K1 (a loss-of-function mutant in the cohesin complex) fission yeast cells. To exclude the cell-cycle effects shown in the rad21-K1 mutant at the restrictive temperature $\left(37^{\circ} \mathrm{C}\right)$ (Tatebayashi et al., 1998), we conducted the Hi-C experiments in semi-permissive temperature $\left(32^{\circ} \mathrm{C}\right)$, as we did for $p f s 2-11$ cells. The Hi-C experiments were performed in two biological replicates and showed high reproducibility of contact distribution according to the Pearson correlation coefficient (Figure 3 - figure supplement 1A) and of Hi-C interaction matrices using HiCRep (Figure 3 - figure supplement 1B). Consistent with previous studies in fission yeast (Mizuguchi et al., 2015, 2014), we observed centromere-proximal arm-arm interactions and Rabl chromosome configuration (Dong and Jiang, 1998; Funabiki et al., 1993; Jin et al., 2000), which is a polarized array of interphase chromosomes such as centromere clustering, in wt cells (Figure 3 - figure supplement 1C). We also confirmed, consistent with previous finding (Mizuguchi et al., 2014), that the interaction domains observed in wt cells were abolished in rad21-K1 cells (Figure 3A, Figure 3 - figure supplement 2A). Together, these results confirm the central role of cohesin in shaping global chromosome territories in fission yeast and validate our application of $\mathrm{Hi}-\mathrm{C}$.

In our Hi-C analysis of rrp6 $\Delta$ and $p f s 2-11$ cells, we observed that similar to the rad $21-K 1$ cells, there was a significant decrease in intra-chromosomal (cis) arm interactions (Figure 3A, Figure 3 figure supplement 2A). Next, we compared the contact probability decay according to the genomic distance, reflecting the general chromatin folding state. The rrp6 $\Delta$ and $p f s 2-11$ mutant cells exhibited a quantitative reduction of short-range interactions within 100-kb (kilobase), with an increase in longrange ( $>300-\mathrm{kb}$ ) interactions (Figure 3B, Figure 3 - figure supplement $2 \mathrm{~B}$ ). Both changes in short- and long-range interactions were reminiscent of the patterns observed in rad21-Kl cells (Figure 3B, Figure 3 - figure supplement 2B). Together, these results demonstrate that 3 '-end RNA processing factors, including Rrp6 and Pfs2, are required to maintain 3D interaction domains and local compaction across the intra-chromosomal arms by modulating cohesin occupancy.

To further dissect how RNA processing factor-associated loss of cohesin binding affects the border formation, we calculated the border strength score, which represents a potential border as previously described (Kim et al., 2016; Van Bortle et al., 2014) whereby the border strength is quantified by a relative ratio of two adjacent intra-domain with inter-domain contact frequencies at a resolution of 3-kb with a window size of 15-kb (Figure 3 - figure supplement 2C). As expected, in wt cells, we detected a high border strength separating the 3D interaction domains (Figure 3C), and it coincided with Rad21 ChIP-seq peaks enriched at converging intergenic sites (Figure 3C-D). Using the border strength, we estimated the size of wt interaction domains in the median size of $65-\mathrm{kb}$, consistent with the previous findings (Mizuguchi et al., 2014; Tanizawa et al., 2017). For further validation, we compared the border strength scores derived from two wt Hi-C datasets (ours and GSM2446268) and observed that both were similarly high at the Rad21 peaks (Figure 3 - figure supplement 2D). We next plotted the border 
strength scores according to the Rad21 levels in wt cells and found a positive correlation between the border strength and Rad21 occupancy (Figure 3 - figure supplement 2E). We also analyzed the published Psc3 (another cohesin subunit) ChIP-chip (GSM1370192) and found that Psc3 was enriched at the maximum border center, defined by the region with maximum border strength scores across the genome (Figure 3 - figure supplement 2F). Thus, these results validate our border strength scores and indicate that strong domain borders at converging intergenic sites are occupied by enriched cohesin.

Using this validated approach for quantifying border strength, we examined domain border strength in $r r p 6 \Delta$ and $p f s 2-11$ cells and detected a significant reduction, similar to what is observed in rad21-K1 cells (Figure 3C,E). This reduction coincided with a decrease in cohesin occupancy (Figure 3D). The degree of border strength changes in each mutant (Figure 3E) indeed corresponded with the changes in Rad21 occupancy at the borders (Figure 3F). To confirm this in a genome-wide fashion, we visualized the Hi-C interactions near Rad21 peaks using insulation distance as previously described (Vara et al., 2019). As expected, we observed that inter-domains (located between adjacent intradomains, Figure 3 - figure supplement 2C) exhibited low Hi-C contacts in wt cells (Figure 3G), suggesting that enriched Rad21 sites insulate adjacent intra-domains. Notably, similar to rad21-K1 cells, we observed increased Hi-C contacts within inter-domains in rrp6 $\Delta$ and $p f s 2-11$ cells, compared to those in wt cells (Figure 2G).

To further examine whether the nuclear exosome indeed affects the 3D chromatin structures, we additionally performed in situ Hi-C using defective mutants in Dis3 (dis3-54), another core and catalytic subunit of the nuclear exosome, with high reproducibility (Figure 3 - figure supplement 3AB). A cold-sensitive mutant of Dis3, dis3-54, shows a reduced RNase activity at its restrictive temperature $\left(20^{\circ} \mathrm{C}\right)$ (Mukarami et al., 2007). As with Pfs2 and Rad21, Dis3 is essential for viability, and thereby we grew dis $3-54$ cells at permissive (wt at $34^{\circ} \mathrm{C}$ ) and semi-permissive temperature (defect at $30^{\circ} \mathrm{C}$ ). Consistent with $\operatorname{rrp} 6 \Delta$ cells, we observed a decrease in intra-chromosomal (cis) arm interactions (Figure 3 - figure supplement 3C) and short-range interactions (Figure 3 - figure supplement 3D) in dis3-54 defect cells, yet albeit weakly due to the use of partially loss-of-function mutant. We compared the changes in the border strength (Figure 3 - figure supplement 3E-F) and the insulation distance analysis (Figure 3 - figure supplement $3 \mathrm{G}$ ) in dis 3-54 wt and mutant cells, suggesting a contribution of Dis3 in the 3D chromatin organizations. Together, these findings support a regulatory role for nuclear exosome in shaping the proper domain borders through the regulation of cohesin positioning.

As Rad21-enriched converging intergenic sites showed discrete domain boundaries, we next wondered whether other Rad21 strongly-enriched loci, including tRNA, rRNA, or promoters, also form cohesin-mediated domain boundaries. However, the intensity of the border strength at each group was relatively smaller than the converging intergenic sites. In particular, tRNA loci distributed across the chromosome arms were strongly enriched with cohesin, and the cohesin localization was dependent on 
nuclear exosome and Cid14 (TRAMP complex) (Figure 1 - figure supplement 1I). Nevertheless, the border strength at the tRNA loci was not as strong as the border strength at converging intergenic sites and was not affected by the deletion of Rrp6 or by rad21-k1 mutant cells (Figure 3 - figure supplement $2 \mathrm{G})$. Therefore, our findings indicate that proper positioning of cohesin at 3'-end of convergent genes via RNA processing factors ensures the formation of discrete and strong domain boundaries.

\section{Proper transcription termination coupled with 3'-end RNA processing is important for the establishment of cohesin at the 3'-end of convergent genes}

Previously, it was suggested that Rad21 is pushed towards the 3'-end of convergent genes by the elongating transcription machinery (Busslinger et al., 2017; Gullerova and Proudfoot, 2008; Heinz et al., 2018; Lengronne et al., 2004). To investigate this in a genome-wide manner, we analyzed transcription levels, distribution of Rad21 and RNA Pol II Ser2 CTD phosphorylation (Ser2P), and border strength using mRNA-seq, ChIP-seq, and in situ Hi-C. In wt cells, we first sorted 1270 pairs of convergent coding genes by transcription strength and then plotted the distribution of Ser2P and Rad21 along with border strength in the same order (Figure 4A, Figure 4 - figure supplement 1A). We observed a strong enrichment of Ser2P and Rad21, coinciding with high border strength (Figure 4A), suggesting that $\operatorname{Rad} 21$ accumulation at highly transcribed gene pairs is responsible for forming domain borders in fission yeast.

We considered that RNAPII accumulated in the intergenic region of the convergent genes could be a trace of RNAPII backtracking activity and a recent study also reported that backtracking of RNA polymerase induces the accumulation of condensin (Rivosecchi et al., 2021). Consistent with a previous study in mammalian cells (Busslinger et al., 2017), at symmetrically expressed sites, Rad21 was positioned symmetrically, and this correlated with the accumulated Ser2P signals at the same converging intergenic sites. Given that the regions we were interested in were highly transcribed loci and may be interfered with the background immunoprecipitation, we normalized Ser2P signals with mock (notag) immunoprecipitation (De Almeida et al., 2010; Pokholok et al., 2005). Deletion of Rrp6 reduced the elongating RNAPII signals at the 3'-ends and increased transcriptional readthrough (Figure 4B, Figure 4 - figure supplement 1A, see red boxes), which indicated the defects in transcription termination. At asymmetrically expressed pairs of convergent genes, we also found the reduced RNAPII at polyadenylation sites (PAS) and downstream-shifted Ser2P signals (Figure 4C). This indicates that reduced cohesin binding at the 3'-end of convergent genes may be linked to Rrp6-mediated termination defects. Recent reports about the novel role of nuclear exosomes in promoting transcription termination by targeting the 3'-end of RNAs exposed from backtracked RNAPII also supported our findings. 
transcriptionally. We further investigated the enrichment of 3'-end processing machinery (Pcf11, Ysh1/CPSF73, and Rna14/CSTF3) and transcription termination factors (Seb1 and Dhp1/Xrn2) using published ChIP-seq data (Larochelle et al., 2018; Wittmann et al., 2017). We also found that these factors were also co-enriched with Ser2P and cohesin at converging intergenic centers (Figure 4D). We observed that Rad21 levels were decreased proportionally to the level of transcription termination factors in rrp6 $\Delta$ cells, compared to wt cells (Figure 4 - figure supplement 1B-C). Together, these data indicate that cohesin and transcription termination-related factors are enriched at highly transcribed convergent gene pairs, correlated with the strong domain borders.

The transcription factor TFIIS, stimulates the intrinsic cleavage activity of RNAPII, thereby allowing the backtracked RNAPII to resume elongation (Izban and Luse, 1992; Reines, 1992). In TFIISdeleted cells, premature dissociation of RNAPII occurs, resulting in premature termination (Lemay et al., 2014; Sheridan et al., 2019; Sigurdsson et al., 2010). Compared to $\operatorname{rrp} 6 \Delta$, when we deleted Tfs1, we only observed a very slight decrease of $\operatorname{Rad} 21$ enrichment at converging intergenic sites. It is reported that although when TFIIS is absent, an intrinsic cleavage activity of RNAPII slowly allows the reactivation of RNAPII (Sigurdsson et al., 2010). As cohesin is translocated by elongating Pol II from promoters and once it reaches toward the 3'-ends, the interplay between RNAPII backtracking and transcription termination (linked with 3'-end processing machinery and nuclear exosomes) may play a crucial role in stabilizing the cohesin complex.

We further explored how the readthrough transcripts produced by the loss of Rrp6 (Figure 4 figure supplement 1D) affect the localization of Rad21. To see whether there is a negative correlation between readthrough transcripts and cohesin intensity (increased readthrough transcripts may cause a greater decrease in cohesin level), we calculated the termination index (Figure 4 - figure supplement 1E). In rrp6 cells, we found that changes in Rad21 level in converging intergenic regions showed no negative correlations with the changes in the level of transcription readthrough produced from the pair of convergent genes (Figure 4 - figure supplement 1F). We conclude that the readthrough transcripts produced from defective RNA processing factors do not solely detach cohesin from the binding sites.

\section{Cohesin is redistributed to facultative heterochromatin at retrotransposon upon Rrp6 depletion}

We revisited the $\operatorname{Rad} 21$ changes upon deletion of $\operatorname{Rrp} 6$ to see whether $\operatorname{Rad} 21$ is redistributed across the genome. We merged 701 Rad21 peaks from 875 and 719 Rad 21 peaks in wt and rrp6 $\Delta$ cells, respectively. Interestingly, we observed 123 increased/newly-created Rad21 peaks (17\%) in rrp6 $\Delta$ cells, which were nearly non-detectable in wt cells (Figure 5A, a bold red line). Furthermore, $51 \%$ of these new Rad 21 peaks were localized in gene bodies (Figure 5 - figure supplement $1 \mathrm{~A}$ ), where $80 \%$ of these genes were either Tf2 retrotransposon elements or proximal to these elements. In S. pombe, there is only 
one family of full-length long terminal repeat (LTR) retrotransposon element (Tf2) (Bowen et al., 2003) with an average size of 4-kb. At all 13 Tf2 elements, we detected substantially increased Rad21 levels in $\operatorname{rrp} 6 \Delta$ cells (Figure 5B, left), while Rad21 levels did not change in pfs2-11 cells, compared to wt

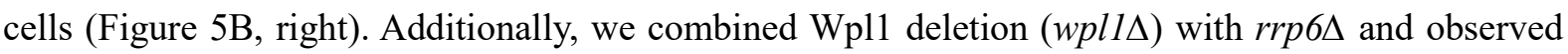

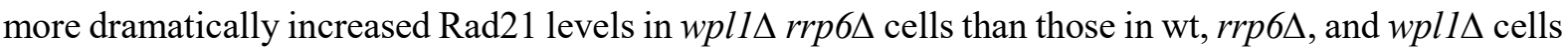
(Figure 5 - figure supplement 1B), confirming that Rrp6/Wp11 independently regulates cohesin positioning. Thus, these data indicate that upon Rrp6 deletion, cohesin is redistributed to specific genomic loci (Tf2).

Recent studies reported RNAi-dependent heterochromatin domain (HOOD) formation at repetitive DNA elements such as centromeric repeats and retrotransposons, and its formation is closely associated with Rrp6. In the absence of Rrp6, which is also known for processing/silencing heterochromatic transcripts, accumulation of siRNA and heterochromatin modifications (H3K9 methylation) were observed. To elucidate whether Rad21 redistribution at Tf2 upon Rrp6 deletion follows the same process as $\operatorname{Rad} 21$ recruitment at heterochromatin (Bernard et al., 2001; Nonaka et al., 2002; Reyes-Turcu et al., 2010), we performed H3K9me3 ChIP-seq and small RNA-seq in wt and rrp6 cells. Indeed, we observed elevated levels of small RNA and H3K9me3 at all Tf2 elements in rrp6s cells compared to wt cells (Figure 5C-D), consistent with previous findings (Yamanaka et al., 2013). Notably, these elevated small RNA and H3K9me3 levels coincided with Rad21 recruitment at specific Tf2 loci in rrp6 cells (Figure 5E). Among 32 defined HOOD loci, all 13 Tf2 are included within HOOD regions, yet Rad21 distribution and heterochromatin markers (small RNA and H3K9me3) on Tf2-excluded remaining HOOD regions were relatively unaffected in $\operatorname{rrp} 6 \Delta$ (data not shown). Furthermore, published H3K9me2 ChIP-seq (GSE114540) (Parsa et al., 2018) showed no notable increase in $p f s 2-11$ mutant compared to wild-type (Figure 5 - figure supplement $1 C$ ). This implies that the production of small RNAs upon Rrp6 deletion induces RNAi-mediated facultative heterochromatin formation on retrotransposon and therefore redistributes Rad21 to these loci in an indirect manner.

We then asked whether Rad21-enriched Tf2 elements could function as a domain border in rrp6 6 cells. To this end, we calculated the border strength at Tf2 elements in wt, $p f s 2-11$, and rrp6 $\Delta$ cells. Importantly, we found increased border strength at Tf2 loci, enriched with Rad21, H3K9me3, and small RNA, in rrp6 $\Delta$ cells compared to wt cells (Figure 5F). In contrast, loss of Pfs2 (pfs2-11) did not affect the formation of domain border (Figure 5G-H), probably due to unchanged Rad21 levels (Figure $5 B, E)$ at Tf2 elements. Therefore, we concluded that Rrp6 is essential for establishing the domain borders and ensuring genome stability by regulating proper cohesin positioning. 
The role of $\operatorname{Rrp6}^{\mathrm{Exosc10}}$ in regulating cohesin and 3D chromatin structures observed in $S$. pombe is similarly conserved in mESC

The functions of the exosome and the cohesin complex are highly conserved from yeast to human. To examine whether the role of the nuclear exosome in $3 \mathrm{D}$ genome organization is conserved in the mammalian genome, we performed in situ Hi-C using mouse embryonic stem cells (mESCs) with RNAi-mediated knockdown of relevant exosome subunit $($ Exosc10 Rrp6) and cohesin subunit $(\operatorname{Rad} 21)$ (Figure 6 - figure supplement 1A). Hi-C analysis achieved high reproducibility between two biological replicates (Figure 6 - figure supplement 1B). Interestingly, Rad21 knockdown (Rad21KD) perturbed the global 3D genome organization in a manner that was consistent with our results obtained in $S . p o m b e$, but knockdown of Exosc10 (Exosc10KD) only marginally affected global chromatin organization compared to that in Egfp knockdown (Control) cells (Figure 6A, Figure 6 - figure supplement 1C-E). Specifically, we observed that markers of global chromatin organization, including the relative contact probability (Figure 6A), the frequency of cis-interactions (Figure 6 - figure supplement 1C), and the number of contact domains/chromatin loops (Supplemental table 3), were disrupted in Rad21KD cells, but not in Exosc10KD cells, compared to Control cells.

To further investigate the roles of Exosc10 and Rad21 in local chromatin organizations, we performed ATA/APA (Aggregate TAD/Peak Analysis) at the Control TADs/loops. Importantly, the ATA/APA results showed a gradual decrease in interactions within TADs (Figure 6B) and chromatin loops (Figure 6C, Figure 6 - figure supplement 1F) from Control in Exosc10KD and Rad2 1KD cells, consistent with our results obtained in S. pombe (Figure 3). Since CTCF (which is absent in yeast) is known to mediate transcriptional insulation, co-localize with cohesin, and contribute to $\operatorname{Rad} 21$ positioning (Parelho et al., 2008; Wendt et al., 2008), we performed ChIP-seq of Rad21 and CTCF in Exosc 10KD mESCs to determine the basis of the gradual decrease in local interactions. In these cells, we observed a slight decrease in Rad21 occupancy at CTCF peaks near TAD boundaries $(P$ value $=$ 0.006), whereas the CTCF peaks showed marginal changes (Figure 6D, Figure 6 - figure supplement 1G). Interestingly, we observed reduced Rad21 occupancy irrespective of CTCF presence (Figure 6D, Figure 6 - figure supplement 6G), implying that even in mammalian genomes, Exosc10 can partially regulate the cohesin occupancy. Finally, we found that the local interactions within the Control TAD were gradually decreased in both Exosc $10 \mathrm{KD}$ and Rad21KD cells, as confirmed by the ATA/APA results. Furthermore, we observed that the reduction of local TAD interactions coincided with the decreased Rad21 enrichment in Exosc10KD cells. These results indicate that Exosc10 plays an important role in organizing the $3 \mathrm{D}$ genome architecture by regulating the $\operatorname{Rad} 21$ localization, and this role is highly conserved from pre-metazoans to metazoans. 


\section{Discussion}

In this study, we used various proteins related to RNA surveillance to examine their role in regulating cohesin complex and $3 \mathrm{D}$ chromatin organization. Using in-situ Hi-C, we observed that ablation of RNA processing factors, including nuclear exosome and Pfs2 (CPF complex), resulted in the loss of cohesin binding and disturbed cohesin-mediated domain boundaries. These results highlight the importance of RNA processing coupled with termination in ensuring proper cohesin positioning at the domain borders where convergent genes mainly exist, thereby maintaining 3D chromatin organizations. Strictly regulated 3'-end RNA processing coupled with proper termination and 3D genome architecture are essential factors for gene expression. This study proved that two processes, which were thought to be independent of each other during transcription, were closely intertwined.

In fission yeast, the cohesin complex is preferentially enriched ( nearly $47 \%)$ at the 3 '-end of convergent genes. 3D domain borders mainly reside at the 3 'end of highly transcribed convergent genes where cohesin and transcription termination factors were co-enriched. Although the intensity of cohesin binding at other cohesin-enriched loci such as tRNA was reduced in RNA processing defective mutants, those regions did not harbor discrete and strong domain borders. Moreover, the border strength at those regions was not dependent on RNA processing factors. These findings once again highlighted the importance of well-positioned cohesin complex at converging interenic sites. In particular, defects in Rrp6 caused Rad21 redistribution to retrotransposon in an indirect manner, resulting in improper domain borders.

At converging intergenic sites, elongating polymerase from both sides continuously approaches toward the 3'-ends, and we observed the accumulation of elongating Pol II (Ser2P) at the end (Figure 4), which may be resulted from the backtracking. Defect in Pfs2, which causes extensive transcription readthrough, prevents backtracked RNAPII. Nuclear exosome targets the 3'-ends of unprotected RNAs that are produced by backtracked RNAPII and promotes transcription termination. Defects in nuclear exosome or extensive transcription readthrough prevent/escape backtracked RNAPII. Recently, a study in fission yeast reported that the RNA polymerase backtracking at gene termini might contribute to the accumulation of condensin, another SMC complex (Rivosecchi et al., 2021). Nevertheless, reduced cohesin binding upon defective 3'-end RNA processing (e.g., rrp6 $\Delta$ or $p f s 2-11$ ) are not solely caused by passive translocation of RNAPII, as we can see in $t f s 1 \Delta$. Taken together, these may explain the active role of RNA processing factors in concert with proper transcription termination in stabilizing the cohesin complex at the 3 'end of genes.

We assumed that the production of readthrough transcripts in $\operatorname{rrp} 6 \Delta$ might explain the decrease of cohesin binding at chromatin. However, we could not find a direct relationship between readthrough transcript and chromatin localization of cohesin in which higher levels of readthrough transcripts 
produced in $\operatorname{rrp} 6 \Delta$ do not always result in cohesin reduction at converging intergenic sites. However, it is still possible that even small amounts of readthrough transcripts may disturb cohesin positioning. For instance, Xist RNA, which regulates the silencing of one of two X chromosomes via acting as a scaffold protein, binds with cohesin and causes repulsion of the cohesin from the inactivated $\mathrm{X}$ chromosome (Minajigi et al., 2015). SA1 and SA2, subunits of the cohesin complex, were reported to bind with various kinds of RNA substrates such as ssRNA, dsRNA and RNA:DNA hybrids (Pan et al., 2021). These evidence leaves a possibility that readthrough transcripts may play a role in regulating cohesin localization.

Furthermore, our results may provide a clue to solving the mystery of chromosomal segregation defects. Previous studies have found chromosomal segregation defects in cells mutant for transcription termination and RNA processing factors, but the underlying mechanisms connecting these have not been resolved (Graham et al., 2009; Mukarami et al., 2007; Wang et al., 2005). A recent study showed that in budding yeast, convergent genes exist at pericentromeres and act as a border that captures cohesin (Paldi et al., 2020). These findings suggest that proper cohesin localization at the pericentromeres may be necessary for chromosome segregation. Our results showed that Rrp6 deletion causes three events: 1) reduction in cohesin occupancy at the 3'-end of convergent genes, 2) increased transcriptional readthrough (defects in transcription termination), and 3) weakness in chromosome arm cohesion. Regarding these, our data suggest that Rrp6-mediated transcription termination and cohesin positioning may play an crucial role in genome integrity (chromosomal segregation).

Influenza A virus (IAV) infection induces global RNAPII transcription termination defects (Zhao et al., 2018). Influenza NS1 protein, in particular, targets the 3'-end processing machinery (CPSF30) and inhibits PAS-dependent termination. As such, IAV infection causes readthrough transcription, disrupts cohesin binding, and therefore results in chromatin decompaction. Therefore, in agreement with recent findings, our results extend the role of RNA surveillance to control the dynamics of cohesin biology and chromatin architecture. We hope that our findings will contribute to the elucidation of cohesin-mediated regulation of 3D chromatin structures, with potential implications for understanding cohesin disruptions in human cancers and cohesinophaties. 


\section{Acknowledgements}

We would like to thank M. Yanagida, K. Ekwall, C.J. Norbury and R. Allshire for providing fission yeast strains. This work was supported by a National Research Foundation of Korea Grant funded by the Ministry of Science and ICT (MSIT) (2018R1A5A1024261, SRC), and the Collaborative Genome Program for Fostering New Post-Genome Industry of the National Research Foundation (NRF) funded by the MSIT (2018M3C9A6065070).

\section{Author Contributions}

Y. Choi, Y. Chun and D. Lee devised the concepts and designed the experiments. S. Han, T. Kim and Y. Chun analyzed the NGS data. Y. Choi and Y. Chun performed most of the experiments, including Hi-C and ChIP-seq. S. Han, and Y. Chun wrote the manuscript under the mentorship and technical supervision of D. Lee and Y. Choi.

\section{Declaration of Interests}

The authors declare that they have no conflicts of interest.

\section{Supplemental Information}

Supplemental Information is available for this paper. 
Figure 1. 3'-end RNA processing factors regulate the localization of the cohesin complex at the 3'ends of convergent genes in euchromatin.

(A) $\operatorname{Rad} 21 \mathrm{ChIP}$ analysis at the 3'-end of convergent genes (wis4-rpl3001 and cdc42-pss 1) in rrp6 4 , dis 3-54 $\left(30^{\circ} \mathrm{C}\right)$, and $p f s 2-11\left(32^{\circ} \mathrm{C}\right)$ compared to wild-type (wt). Data for Rad21-5XFLAG ChIP and mock (non-tagged control; notag) are presented as RPM values. RPM, Reads Per Million mapped reads. (B) Metaplot (top) and heatmap (bottom) analysis of Rad21 ChIP signals at convergent intergenic regions $(n=292)$ in indicated mutants. $(C)$ Box plots showing normalized Rad21 intensity $(\operatorname{Rad} 21$ IP normalized by mock) for the regions $\pm 300 \mathrm{bp}$ from the $\operatorname{Rad} 21$ peaks as in (B). (D) Genome browser showing a comparison of $\operatorname{Rad} 21$ distribution along a 3,310 - 3,520 kb region of chromosome II. Only convergent gene pairs (forward-strand gene, blue box; reverse-strand gene, red box) overlapped with $\operatorname{Rad} 21$ peaks are indicated below. Box plots represent the median (center bold line), 25th and 75th percentiles (box plot boundaries), and the whiskers extending to $\pm 1.5 \times$ interquartile range (IQR).

Figure 2. Rrp6 directly regulates cohesin occupancy.

(A) Scheme of a tetracycline-regulated system for temporally controlled regulation of $\mathrm{rrp}^{+}$gene expression; ahTet indicates the tetracycline analog, anhydrotetracycline. (B) Western blot validating the successful knockdown and rescue of FLAG-tagged Rrp6 expression. (C, D) Heatmaps (C) showing the distribution of normalized Rad21 signals ( $\operatorname{Rad} 21$ IP normalized by mock) across a time-course of ahTet withdrawal and treatment, and representative genome browser results (D) showing Rrp6-dependent changes in Rad21 occupancy. (E) Box plot showing normalized Rad21 intensity (Rad21 IP normalized by mock) changes across a time-course of ahTet withdrawal and treatment. (F, G) Metaplot (F) and genome browser results (G) showing Rrp6- and Wpl1-dependent changes of Rad21 occupancy at converging intergenic regions $(n=292)$. (H) Representative genome browser results showing Rrp6/Wpl1 dependent changes of Rad21 at convergent gene pairs.

Figure 3. Nuclear exosome and Pfs2 regulate the higher-order 3D genome architecture via rad21.

(A) In situ Hi-C map (3-kb resolution) showing the Hi-C interactions in chromosome III (top) and the $1,200-1,700 \mathrm{~kb}$ region of chromosome III (middle) of wt, $\operatorname{rad} 21-K 1, \operatorname{rrp} 6 \Delta$, and $p f s 2-11$. The relative Hi-C map (bottom) comparing mutants ( $r a d 21-K 1$, rrp6 $\Delta$, and $p f s 2-11$ ) with wt was calculated by subtraction (mutant - wild type). Arrows indicate notable changes in the interaction domains. (B) Relative contact probability (RCP) plot (top) showing contact frequency relative to genomic distance. Box plots (bottom) comparing the contact probabilities of short-range $(<100-\mathrm{kb})$ and long-range $(>$ $300-\mathrm{kb})$ interactions in indicated mutants. $P$ values were calculated using the paired Wilcoxon ranksum test. (C) Representative Hi-C map showing the 1,200 - 1,700 kb region of chromosome III (3-kb resolution). Border Strength is calculated with $3-\mathrm{kb}$ resolution of $15-\mathrm{kb}$ window sizes. See also Figure 3 - figure supplement 2C. (D) Rad21 ChIP-seq (RPM) of wt, rrp6 4 , and $p f s 2-11$ track is aligned with the above Hi-C map (C). In particular, sites of reduced border strength along with reduced Rad21 peaks upon defects in Rrp6 or Pfs 2 are marked with asterisks. The lower panels illustrate a zoomed-in view of Rad21 ChIP. (E) Metaplot (Left) and boxplot (Right) of border strength at Rad21 peaks at converging intergenic sites. (F) Enrichment of $\operatorname{Rad} 21 \mathrm{ChIP}$ at the center of domain border in wt, $r r p 6 \Delta$, and $p f s 2-$ 11 mutants. (G) Aggregate plots generated from Hi-C data for Rad21 peaks $(\mathrm{n}=292)$ showing discrete cohesin-mediated border. Box plots represent the median (center bold line), 25th and 75th percentiles 
(box plot boundaries), and the whiskers extending to $\pm 1.5 \times$ interquartile range (IQR).

\section{Figure 4. Proper transcription termination coupled with 3 '-end processing is important for the establishment of cohesin at the 3 '-end of convergent genes.}

(A) Heatmaps sorted by the median mRNA expression (RPKM) of total convergent gene pairs. ChIPseq of Rad21 (RPM) and RNAPII Ser2P phosphorylation (Ser2P) and the border strength were plotted against the total converging intergenic center. (B, C) Metaplot analysis of Rad21 (RPM) and Ser2P (normalized by FLAG-immunoprecipitated non-tagged control; notag) occupancy at either symmetrically (B) or asymmetrically (C) expressed pairs of convergent genes. Converging gene pairs with symmetric (mRNA RPKM difference between the gene pairs is less than 2-fold) or asymmetric transcription strength are sorted by mRNA RPKM and divided into either three groups (highly expressed $(n=148) ; 0-35 \%$, moderately expressed $(n=152) ; 35-70 \%$, and lowly expressed $(n=130)$; $70-100 \%$ ) (B) or two groups (forward convergent genes strongly transcribed than reverse convergent genes $(n=190)$ (Left) and vice versa (Right) $(n=249))(C)$. Blue dotted lines indicate the average Rad21 enrichment center. (D) Heatmaps sorted by the same order as in (A) showing the co-localization of Rad21 and Ser2P with termination factors (Dhp1, Seb1) and CPSF complex (Pcf11, Rna14, and Ysh1) at highly transcribed converging genes. Transcription termination factors (Pcf11, Seb1, Rna14, Ysh1, Dhp1) were derived from published ChIP-seq data (GSE93344 and GSE 115595). (E, F) Metaplot (E) and box plot (F) of Rad21 peaks (normalized by mock) in indicated mutant cells. (G) Representative genome browser results showing $\operatorname{Rad} 21$ enrichment in indicated mutants (tfs $1 \Delta$ and $\operatorname{rrp} 6 \Delta$ ) at convergent gene pairs.

\section{Figure 5. Cohesin is redistributed to facultative heterochromatin at retrotransposon upon Rrp6 depletion.}

(A) Heatmaps of Rad21 ChIP-seq signals (RPM) at combined Rad21 peaks at both wt and rrp6 6 , sorted by change (rrp6 $\Delta / \mathrm{wt})$ in $\operatorname{Rad} 21$ intensity. Assignment of Rad21 peaks that overlap or proximal to either convergent intergenic regions (Convergent Int) or Retrotransposon ( $\mathrm{Tf} 2$ elements) are shown on the right panel. (B) Metaplots of Rad21 (RPM) binding of wt, $r r p 6 \Delta$, and $p f s 2-11$ at 13 retrotransposon elements. (C, D) Normalized (forward or reverse) small RNA reads of wt and rrp6 $(\mathrm{C})$ and H3K9me3 $\left(\log _{2} r r p 6 \Delta / w t\right)(D)$ at 13 retrotransposon elements. (E) Genome browser example of chromatin features at retrotransposon element Tf2-7-Tf2-8 where Rad21 binding is increased upon Rrp6 deletion. (F) In situ Hi-C map (5-kb resolution) (top) and the border strength (middle) and chromatin features (bottom) at retrotransposon element Tf2-3. (G) Average profile of border strength plotted against the center of 13 retrotransposon elements. (H) Box plot showing significantly increased border strength at the center of 13 retrotransposon elements in $\operatorname{rrp} 6 \Delta$ compared to wt and $p f s 2-11$.

\section{Figure 6. The role of $\operatorname{Rrp6}^{\text {Exosc10 }}$ in regulating cohesin and 3D chromatin structures observed in $S$. pombe is similarly conserved in mESC.}

(A) Relative contact probability (RCP) plot showing contact frequency relative to genomic distance. The dashed box displays the size range of TADs $(\leq 1 \mathrm{Mb})$. (B) Aggregate TAD analysis (ATA) comparing interactions of Exosc $10 K D$ and Rad2 $1 K D$ with Control $(E g f p K D)$ cells. Control TADs were used for ATA ( $\mathrm{n}=4508)$. (C) Aggregate peak analysis (APA) comparing Control, Exosc10KD, and Rad21KD cells. Control peaks (chromatin loops) were used for APA $(\mathrm{n}=12,477)$. (D) Example of a TAD that lost its interactions (dashed box with blue arrow) upon Exosc10 and Rad21 knockdown. HiC contact maps (top), insulation heatmaps (middle), and Rad21 and CTCF ChIP-seq peaks (bottom; red 
and blue bars, respectively) are shown for Control, Exosc10KD, and Rad21KD mESCs. The ChIP-seq tracks display zoom-in browser view of dashed box showing where a TAD loses its interactions. The decreased Rad21 ChIP-seq peaks upon Exosc10KD are marked with asterisks.

\section{Figure supplements}

\section{Figure 1 - figure supplement 1}

(A) Classification of $\operatorname{Rad} 21$ binding $(\mathrm{n}=867)$ sites at euchromatin. 'Tandem intergenic' indicates the intergenic regions between a pair of tandemly oriented genes. (B) Western blot validating the successful knockdown of Dis3 expression. Knockdown of Dis3 was achieved by incubating cells at rich medium (without anhydrotetracycline) for 10 hours. Ponceau S staining was used for the confirmation of equal loading. (C) RT-qPCR analysis showing the readthrough transcription at pss 1 locus by reversetranscribing with a gene-specific primer (pss 1 probe) which targets downstream of Polyadenylation site in wt (0hr) and knockdown (10hr) of Dis3. (D) ChIP-qPCR analysis of Rad21 intensity upon knockdown (10hr) of Dis3. 5'UTR region of rps001 was used as a negative control. Three biological replicates were used for (C) and (D). P values were calculated using the two-paired student t-test. (E, F) Representative examples (E) and box plot analysis (F) of Psc3 occupancy enriched at the 3'-end of convergent genes in wt and $\operatorname{rrp} 6 \Delta$. (G, H) Representative examples $(\mathrm{G})$ and box plot analysis $(\mathrm{H})$ of Rad21 occupancy enriched at the 3'-end of convergent genes in indicated strains. Each ChIP signal from box plot analysis (F, H) was normalized to that of the non-tagged control. (I) Metaplot analysis of Rad21 ChIP signals at indicated regions in various RNA processing mutants.

\section{Figure 1 - figure supplement 2}

(A) Genome browser showing Rad21 ChIP-seq results at the centromere and telomere (right arm) of chromosome I. (B) Protein expression of FLAG-tagged Rad21 was unaffected in mutants, as assessed by Western blot analysis. Ponceau-S staining was used for the confirmation of equal loading.

\section{Figure 2 - figure supplement 1}

(A) Protein expression of FLAG-tagged Rad21 was unchanged in rrp64, wpl14, and rrp64 wpl14 when compared to wt. Ponceau-S staining was used for the confirmation of equal loading. (B) GFP spots indicating LacI-GFP bound to $\mathrm{LacO}$ repeats inserted at $a d e 6^{+}$site, which resides in chromosomal arm region. Merged GFP and DIC images are shown. (C) Bar plot shows the percentage of cells with two separate GFP foci from among more than 100 cells. GFP spots were counted from images captured at $0.3-\mu \mathrm{m}$ intervals in the z-axis. (D) A spotting assay showing that Rrp6 genetically interacts with Swi6.

\section{Figure 3 - figure supplement 1}

(A) Scatter plots showing high reproducibility among the replicates of all $\mathrm{Hi}-\mathrm{C}$ samples, as assessed using contact probability based on Pearson correlation. (B) A stratum adjusted correlation coefficient (SCC) was used to compare the similiary across the replicate samples and each mutant using ICEnormalized Hi-C interaction matrices. (C) Genome-wide in situ Hi-C map of wild-type cells at 3-kb resolution showing the Rabl configuration. Dashed circles inside the Hi-C map indicate cen-cen and dashed triangles indicate tel-tel interactions.

\section{Figure 3 - figure supplement 2}

(A) In situ Hi-C map (3-kb resolution) in wt, $r r p 6 \Delta$, pfs 2-11, and rad21-K1 showing the interaction in 
chromosome II (top) and the 2,500 - 2,900 kb region of chromosome II (bottom). (B) Relative contact probability (RCP) plot showing contact frequency relative to genomic distance. The dashed boxes display short-range $(<100-\mathrm{kb})$ and long-range $(>300-\mathrm{kb})$ interactions. (C) Scheme of border strength calculation (Van Bortle et al., 2014). (D) Enrichment of border strength calculated from this study (wt) and published data (GSM2446268) on Rad21 peaks that reside in converging intergenic sites. (E) Metaplot analysis of border strength according to the $\operatorname{Rad} 21$ peaks grouped by $\operatorname{Rad} 21$ ChIP intensity. (F) Enrichment of Psc3 ChIP-chip (GSM1370192) at the center of border in wt. (G) Metaplot analysis of the border strength at Rad21-enriched loci. Each plot was plotted against the center of Rad21 peaks overlapping with annotated regions. A purple dotted line indicates the border strength profile at convergent intergenic sites.

\section{Figure 3 - figure supplement 3}

(A) Scatter plots showing high reproducibility in dis3-54 cells using Pearson correlation analysis as in Figure 3 - figure supplement 1A. (B) A stratum adjusted correlation coefficient (SCC) was used to compare the similiary across the replicate samples and each mutant using ICE-normalized Hi-C interaction matrices. (C) In situ Hi-C map (3-kb resolution) in dis3-54 mutants showing the interaction in chromosome III (top) and the 1,200 - 1,700 kb region of chromosome III (bottom). A relative Hi-C map comparing the mutants with that of wt was calculated by subtraction. (D) RCP plot showing contact frequency relative to genomic distance. The dashed boxes display short-range $(<100-\mathrm{kb})$ and long-range $(>300-\mathrm{kb})$ interactions. (E) Representative Hi-C map showing the 1,200 - 1,700 kb region of chromosome III (3-kb resolution). Border Strength is calculated with 3-kb resolution of $15-\mathrm{kb}$ window sizes. Arrows indicate notable changes in the interaction domains. (F) Metaplot (Left) and boxplot (Right) of border strength at Rad21 peaks at converging intergenic sites (G) Aggregate plots generated from Hi-C data in dis3-54 mutants at convergent gene pairs enriched with strong Rad21 peaks $(\mathrm{n}=292)$ showing cohesin-mediated border boundary.

\section{Figure 4 - figure supplement 1}

(A) Representative examples of genome browser views showing shifted Ser2P and decreased Rad21 ChIP intensity along with increased readthrough transcripts in rrp6 $\Delta$ (dotted red box) (B) Comparison of Rad21 enrichment level according to five groups sorted by the level of Seb1 (A, top) and Pcf11 (A, bottom) at $\operatorname{Rad} 21$ peaks at convergent intergenic regions. (C) $\log 2$ fold change of $\operatorname{Rad} 21$ (rrp6 $\Delta / \mathrm{wt})$ were plotted by the aforementioned groups in (B). (D) Metaplot of mRNA-seq signals downstream of PAS against Rad21-enriched convergent genes. (E) To quantitatively compare termination defects (in terms of readthrough transcript) in rrp $6 \Delta$ with wt, we used termination index to indicate the readthrough intensity. Termination index (TI) is determined by dividing B (the read count within PAS +500 bp) by A (the read count within PAS - 500bp). PAS, Polyadenylation signal. (F) Scatter plot comparing the change in readthrough intensity (calculated as the termination index) as $\log _{2}$ (rrp6 $\Delta / \mathrm{wt}$ ) (x-axis) with that of $\operatorname{Rad} 21$ occupancy as $r r p 6 \Delta / \mathrm{wt}$ (y-axis).

\section{Figure 5 - figure supplement 1}

(A) Annotation of increased Rad 21 peaks upon Rrp6 deletion. (B) Heatmaps of Rad21 ChIP-seq signals (RPM) in indicated strains at 13 retrotransposon elements. Relative fold change of Rad21 signals in rrp $6 \Delta$ and rrp $6 \Delta$ wpll $\Delta$ compare to wt was compared by calculating median Rad 21 intensity at $13 \mathrm{Tf} 2$ ORFs. (C) Metaplot analysis comparing H3K9me2 levels in $p f s 2-11$ mutant ( $2 \mathrm{hr}$ heat shock at $37^{\circ} \mathrm{C}$ ) 
and wt state. The H3K9me2 data were derived from the published dataset (GSE114535). (D) In situ HiC map (5-kb resolution) (top), the border strength (middle), and chromatin features (bottom) at retrotransposon element $T f 2-12$ at wt and $\operatorname{rrp} 6 \Delta$.

\section{Figure 6 - figure supplement 1}

670

671

672

673

674

675

676

677

678

679

680

681

682

683

684

685

686

687

688

(A) Bar plot showing the knockdown efficiencies of siRNA against Exosc10 and Rad21. The levels of the target genes (Exosc10 and Rad21) were normalized relative to that of Act1. Data are presented as mean \pm SD. (B) Density plots showing the frequency of cis interactions of two replicates in Control (left), Exosc10KD (middle), and Rad21KD (right) mESCs. Black and red dotted lines indicate replicates 1 and 2, respectively. (C) Density plot showing the frequency of cis interactions of Control (black line), Exosc10KD (red dotted line), and Rad21KD (light blue line) mESCs. (D) Scatter plots comparing genome-wide PC1 (first principal components, equivalent to the first eigenvectors) values between Exosc10KD (left, Pearson $\mathrm{R}^{2}=0.991$ ) and Rad21KD (right, Pearson $\mathrm{R}^{2}=0.977$ ) with Control cells. (E) Cumulative distribution of TAD sizes in Control (gray dots), Exosc10KD (red dots), and Rad21KD (light blue dots) mESCs. The dashed line indicates average TAD size. (F) Box plot displaying contacts (interactions) of Control (gray), Exosc10KD (red), and Rad21KD (light blue) mESCs at Control peaks (chromatin loops). The horizontal line in the box denotes the median. $P$ values were calculated using the paired Wilcoxon rank-sum test $\left(* * * \mathrm{P}<10^{-100} ; * * \mathrm{P}<10^{-50}\right)$. (G) Example of a TAD that lost its interactions (dashed box with blue arrow) upon Exosc10 and Rad21 knockdown. Hi-C contact maps (top), insulation heatmaps (middle), and Rad21 and CTCF ChIP-seq peaks (bottom, red and blue bars, respectively) are shown for Control, Exosc10KD, and Rad21KD ESCs. The ChIP-seq tracks display zoom-in browser view of the dashed box, showing where a TAD loses its interactions. The decreased Rad21 ChIP-seq peaks upon Exosc10KD are marked with asterisks. 
Materials and Methods

690

\section{Yeast strains and culture}

The strains used in this study are listed in Supplemental Table 1. All deletion strains and tagged strains were constructed by gene targeting using PCR-based methods (Bähler et al., 1998). Yeast cells were grown in YES (Yeast-Extract with Supplements) medium at $30^{\circ} \mathrm{C}$. The temperature-sensitive mutants, including rad21-K1 $\left(32^{\circ} \mathrm{C}\right)$, pfs $2-11\left(32^{\circ} \mathrm{C}\right)$, and dis $3-54\left(30^{\circ} \mathrm{C}\right)$ were cultured at their semi-permissive temperatures. For the in situ $\mathrm{Hi}-\mathrm{C}$ experiments, yeast cells were grown at $32^{\circ} \mathrm{C}$ unless otherwise indicated.

For $\operatorname{dis} 3^{+}$and $r r p 6^{+}$gene knockdown and rescue experiments, their gene promoters were replaced with a tetracycline-responsive promoter harboring TRE (tetracycline response element) (Zilio et al., 2012). Cells were first grown to mid-log phase $\left(\sim \mathrm{OD}_{600} 0.6\right)$ at $30^{\circ} \mathrm{C}$ in YES medium supplied with $2.5 \mu \mathrm{g} / \mathrm{ml}$ of anhydrotetracycline (ahTet). To deplete Dis3 and Rrp6, cells were shifted to YES medium lacking ahTet and incubated for indicated times ( $\sim 10 \mathrm{hr}$ for Dis3 and 8, $12 \mathrm{hr}$ for Rrp6). To recover Rrp6 expression, the cells were then shifted to fresh YES medium containing ahTet $(2.5 \mu \mathrm{g} / \mathrm{ml})$ and incubated for the indicated times ( 0.5 and 4 hours).

\section{Mouse embryonic stem cell culture}

E14Tg2a mESCs were cultured on feeder-free gelatin-coated plates in Glasgow minimum essential medium (GMEM, Gibco) supplemented with 10\% knockout serum replacement (Gibco), 1\% defined fetal bovine serum (FBS, Hyclone), 1\% non-essential amino acids (NEAA, Gibco), 1\% sodium pyruvate (Gibco), 0.5\% antibiotic-antimycotic (penicillin-streptomycin containing, Hyclone), $0.1 \mathrm{mM}$ $\beta$-mercaptoethanol (Gibco), and $1000 \mathrm{U} / \mathrm{ml}$ leukemia inhibitory factor (LIF, Millipore). E14Tg2a $\mathrm{mESCs}$ were grown at $37^{\circ} \mathrm{C}$ in air containing $5 \% \mathrm{CO}_{2}$.

\section{RNA interference}

Silencer ${ }^{\circledR}$ Selects s78573 (5 nmol, Thermo) and Silencer ${ }^{\circledR}$ Select s72659 (5 nmol, Thermo) were used as the siRNAs against Exosc $10^{\mathrm{Rrp} 6}$ and Rad21, respectively, while the control siRNA sequence against EGFP, GUUCAGCGUGUCCGGCGAGUU, was produced by Bioneer (Daejeon, Korea). E14Tg2a mESCs $\left(1 \times 10^{5}\right.$ cells/well) were seeded to 6-well plates. After 24 hours, the cells were transfected with $50 \mathrm{nM}$ of siRNA against Exosc10, Rad21, or Egfp using DharmaFECT 1 (T-2001-03, Dharmacon) and Opti-MEM (Gibco) according to the manufacturer's protocols. At 48 hours post-transfection, the cells were harvested and analyzed by RT-qPCR.

\section{In situ Hi-C}

The in situ Hi-C library construction in fission yeast was performed as previously described (Rao et al., 2014), with slight modifications. Briefly, $2.4 \times 10^{9}$ mid-log phase cells were fixed with $3 \%$ formaldehyde for $15 \mathrm{~min}$ and then quenched with $125 \mathrm{mM}$ glycine. The fixed cells were washed with ice-cold TBS $(20 \mathrm{mM}$ Tris- $\mathrm{HCl} \mathrm{pH} 7.5,150 \mathrm{mM} \mathrm{NaCl}$ ), nuclei were extracted by zymolyase digestion, and $6 \mu \mathrm{g}$ of pelleted nuclei were used for Hi-C library construction. Each nuclear pellet was incubated in $50 \mu \mathrm{l}$ of $0.5 \% \mathrm{SDS}$ at $62^{\circ} \mathrm{C}$ for 10 minutes and then immediately quenched with $170 \mu \mathrm{l}$ of $1.47 \%$ Triton X-100 
at $37^{\circ} \mathrm{C}$ for 15 minutes. Chromatin was digested with 100 units of $\mathrm{MboI}(\mathrm{NEB})$ at $37^{\circ} \mathrm{C}$ for 2 hours and subsequently incubated at $62^{\circ} \mathrm{C}$ for 20 minutes to inactivate the MboI. To fill in the overhangs of restriction fragments and mark the DNA ends, we incubated each sample with $37.5 \mu 1$ of $0.4 \mathrm{mM}$ biotin14-dCTP and $1.5 \mu \mathrm{l}$ of $10 \mathrm{mM}$ dATP, dGTP, and dTTP (all from Life Technologies) plus 40 units of Klenow fragment (NEB) at $23^{\circ} \mathrm{C}$ for 1.5 hours. We then performed ligation with 2,000 units of T4 DNA ligase (NEB) at $23^{\circ} \mathrm{C}$ for 4 hours with slow rotation. After the samples were treated with proteinase $\mathrm{K}$ (NEB) and $10 \%$ SDS, the chromatin was decrosslinked overnight in the presence of $250 \mathrm{mM} \mathrm{NaCl}$ at $68^{\circ} \mathrm{C}$. DNA was purified using AMPure XP beads (Beckman Coulter) and sheared to 300-500 bp using a focused ultrasonicator (Covaris S220). The biotinylated DNA was selectively purified using Dynabeads MyOne Streptavidin T1 beads (Life Technologies). Hi-C library construction (end repair, adenylation, and adapter ligation) of the biotinylated DNA was done manually and adapter ligation was done using a TruSeq DNA Index kit (Illumina). The Hi-C library was quantified using a KAPA library quantification kit (Roche), and further PCR amplification was performed using Phusion Hot Start II DNA polymerase (Thermo Scientific). Finally, the library was sequenced with an Illumina HiSeq 2500 and HiSeq 4000 to obtain either 75-bp or 100-bp paired end reads, respectively.

The in situ Hi-C experiments for Control, Exosc10KD, and Rad21KD mESCs (1 million cells each) were performed according to the Arima-HiC protocol described in the Arima-HiC+ kit (Arima Genomics). The Illumina-compatible Hi-C libraries were prepared according to the Arima-HiC protocol and the library was sequenced with an Illumina Novaseq6000 using the pair-end method (150-bp reads).

\section{In situ $\mathrm{Hi}-\mathrm{C}$ analysis in fission yeast}

Basic Hi-C analysis. The sequenced paired-reads were analyzed using a HiC-Pro (Servant et al., 2015) workflow which is an integrated tool that can be used to preprocess Hi-C data by mapping, filtering, building the interaction matrix, and normalization. Statistics of the Hi-C data are shown in Supplemental Table 2. The raw paired-reads were mapped to the $S$. pombe reference genome (ASM294v2) using bowtie2 (Langmead and Salzberg, 2012) (v2.3.4). Hi-C artifacts (low mapping quality, multi-mapped reads, singletons, dangling end, self-circle, and dumped pairs) were removed and the valid read pairs between samples were then merged. To normalize the difference in sequencing depth from each Hi-C data, the in situ Hi-C data were normalized by the smallest number of valid read pairs. The raw and ICE-normalized matrix was generated at $1-\mathrm{kb}$ and $3-\mathrm{kb}$ bin resolutions using normalized valid pairs. To measure reproducibility among the replicates, we assessed the reproducibility either by comparing the contact probability using pearson correlation method or by measuring the correlation among Hi-C interaction matrices using a stratum-adjusted correlation coefficient (Yang et al., 2017).

Heatmap and plot. All heatmaps were generated based on the 3-kb iced-normalized matrix from HiCPro, using HiCPlotter (Akdemir and Chin, 2015) and HiCExplorer (Ramírez et al., 2018). The contact probability plot was calculated according to the genomic distance, and the quantitative comparisons of short-range $(<100-\mathrm{kb})$ and long-range $(>300-\mathrm{kb})$ contact probability plots were visualized using the ggplot2 package in R. For quantitative comparison of contact probability for each range (short-range and long-range), we used 1-kb resolution to obtain a sufficient number of bins for the analysis. Wilcoxon rank-sum tests were used to measure the statistical significance of each mutant compared to wild-type in terms of the short-range and long-range contact probabilities.

Potential border. To determine the potential border, we calculated the border strength (Kim et al., 2016; Van Bortle et al., 2014) for the $S$. pombe genome. First, we divided the $S$. pombe genome into 3-kb bins. The range within \pm 5 bins ( $=15-\mathrm{kb})$ was set to 'intra-domain' for all bins in the genome, and the domain between upstream intra-domain and downstream intra-domain was termed as 'inter-domain'. The score 
for each domain was calculated by the sum of the iced-normalized interaction score in the domain. The ratio between the two domains was calculated by dividing the intra-domain score by the inter-domain score. All bins were normalized using the z-score. The border strength score can explain the existence of a potential border; a positive strength score means that the interaction within the upstream and downstream intra-domain of a specific bin occurs more frequently than the interaction in the interdomain.

Cohesin-mediated domain boundary. To generate cohesin-mediated meta border plots, the icednormalized matrix was transformed into an obs/exp matrix (Vara et al., 2019). Then, the sub-matrix was extracted for the $\pm 50 \mathrm{~kb}$ region from the center of the convergent gene with strong Rad 21 peaks. After all of the sub-matrices were averaged, the results were transformed with $\log 10$ and used to generate a meta plot via HiCExplorer.

\section{In Situ Hi-C analysis in mouse embryonic stem cells}

Basic Hi-C analysis. Hi-C data were aligned to the mouse genome (mm10) and normalized using HiCPro. Statistics of the Hi-C data are shown in Supplemental Table 2. To achieve maximum sequencing depth, we pooled all replicates from the samples and proceeded to downstream analysis using R package GENOVA unless otherwise stated (https://github.com/robinweide/GENOVA) (Haarhuis et al., 2017). Principal Component Analysis (PCA). PCA was performed using HOMER (Heinz et al., 2018). Briefly, Hi-C paired-end reads were trimmed using HOMER (homerTools trim) and then separately aligned to the mouse genome ( $\mathrm{mm} 10$ ) using bowtie2. Aligned Hi-C data were processed using HOMER (makeTagDirectory, runHiCpca.pl) with 100-kb resolution to achieve principal component 1 (PC1) values.

TAD calling. TAD calling was performed using CaTCH (Zhan et al., 2017). TADs were identified according to the reciprocal insulation (RI) value with the maximum CTCF enrichment at the TAD boundaries.

Contact domain and peak calling. Contact domain and peak calling were performed using JUICER (Durand et al., 2016). Contact domains with resolutions of 10, 20, 40, 50, and 100-kb were identified using the arrowhead algorithm (Rao et al., 2014). Peaks (chromatin loops) were identified using the HiCCUPS algorithm with default options (medium-resolution maps).

Aggregate TAD analysis (ATA). ATA was performed using the R package GENOVA. Briefly, we used $20-\mathrm{kb}$ resolution Hi-C matrices to calculate the average contact frequency matrices in the Control (siEgfp-treated mouse embryonic stem cell) TADs. To account for different TAD sizes, the Hi-C matrices were scaled to the same size, and the 5' and 3' TAD borders were extended to half of the TAD sizes. Finally, the differential ATA was produced by subtracting the average contact frequency matrices of knockdown (Exosc10KD or Rad21KD) and Control cells.

Aggregate peak analysis (APA). APA was performed using the R package GENOVA. Briefly, we used $10-\mathrm{kb}$ resolution Hi-C matrices to calculate the average contact frequency at the Control (siEgfp-treated mouse embryonic stem cell) peaks. The regions surrounding the Control peaks were extended as indicated in the figures.

Insulation score analysis. Insulation score analysis was performed using R package GENOVA. Briefly, genome-wide insulation scores (Crane et al., 2015) were calculated at 20-kb resolution with default options (window size $=25$ ).

\section{Chromatin immunoprecipitation assay and ChIP-seq library preparation}


819 ChIP assays (in fission yeast) were performed as previously described (Strahl-Bolsinger et al., 1997), with slight modifications. For the quantitative comparison of Rad21-5XFLAG ChIP in rrp6 $\Delta$ and wplls background cells, we mixed formaldehyde-fixed $S$. pombe and $S$. cerevisiae at a 19: 1 ratio $\left(\mathrm{OD}_{600}\right.$ ratio) before ChIP cell lysis step. Briefly, lysates were sonicated 12 times (15 sec on, $180 \mathrm{sec}$ off) using a Dismembrator Model 500 sonicator (Fisher Scientific) at 30\% output. The immunoprecipitated DNA was eluted using a SigmaPrep ${ }^{\mathrm{TM}}$ spin column and then subjected to protease treatment and decrosslinking. The extracted DNA was purified using a QIAquick PCR purification kit (Qiagen). The quantification of immunoprecipitated DNA relative to input was done by real-time ChIP-qPCR using Biorad CFX96 with EvaGreen Mix (BioFACT). All Primers used in the ChIP-qPCR were listed in Supplemental Table 4.

ChIP assays in mESCs were performed as previously described (Kim et al., 2014), with minor modifications. Briefly, mESCs were washed with PBS and incubated with $1 \%$ formaldehyde for $10 \mathrm{~min}$ at $25^{\circ} \mathrm{C}$. The crosslinking was quenched with $125 \mathrm{mM}$ glycine for $5 \mathrm{~min}$ at $25^{\circ} \mathrm{C}$, and the cells were harvested with cold PBS. The cells were suspended in SDS lysis buffer $(50 \mathrm{mM}$ Tris-HCl, $\mathrm{pH} 8.0,1 \%$ SDS, $10 \mathrm{mM}$ EDTA) and sheared to mono- or dinucleosome sizes using. The sonicates were incubated overnight with the relevant antibodies and Protein A, G sepharose (17-1279-03 and 17-0618-05, GE Healthcare) at $4^{\circ} \mathrm{C}$ with agitation. The immune complexes were washed for $10 \mathrm{~min}$ each with the following wash buffers: low-salt wash buffer (20 mM Tris-HCl, pH 8.0, $150 \mathrm{mM} \mathrm{NaCl}, 0.1 \% \mathrm{SDS}, 1 \%$ Triton X-100, $2 \mathrm{mM}$ EDTA), high-salt wash buffer (20 mM Tris-HCl, $\mathrm{pH} 8.0,500 \mathrm{mM} \mathrm{NaCl}, 0.1 \%$ SDS, 1\% Triton X-100, 2 mM EDTA), and LiCl wash buffer (10 mM Tris-HCl, pH 8.0, $250 \mathrm{mM} \mathrm{LiCl,}$ $1 \%$ NP-40, $1 \%$ sodium deoxycholate, $1 \mathrm{mM}$ EDTA). The immune complexes were further washed twice with TE buffer (10 mM Tris-HCl, pH 8.0, $1 \mathrm{mM}$ EDTA), eluted with elution buffer (1\% SDS, $0.1 \mathrm{M}$ $\mathrm{NaHCO} 3)$, and decrosslinked overnight at $68^{\circ} \mathrm{C}$. The immunoprecipitated DNA was treated with proteinase $\mathrm{K}$ and RNase A and recovered by phenol-chloroform-isoamyl alcohol precipitation.

Sequencing libraries were prepared using either a NEXTflex ${ }^{\mathrm{TM}}$ Illumina ChIP-Seq Library Prep kit (5143-02, BIOO) or Accel-NGS 2S Plus DNA Library Kit (21024, SWIFT) according to the manufacturer's protocols. Sequencing was performed on a HiSeq2500 using the single-end method (50bp reads) or a Novaseq6000 using the pair-end method (150-bp reads).

\section{ChIP-seq data analysis}

The sequenced reads were mapped to the $S$. pombe genome (ASM294v2) using NovoAlign (Novocraft Technologies) with default parameters. For pair-end reads, only the R1 reads were used for analysis. Bigwigs were created using Deeptools (Ramírez et al., 2016) bamCoverage, with bamfiles normalized to the number of reads per bin by the number of mapped reads in million (RPM). The bigwigs were normalized by mock (non-tagged control). Raw IP data or normalized data were visualized by integrative genome viewer (IGV). Plots and heatmaps of ChIP-seq data were generated using computeMatrix and plotProfile/Heatmap from Deeptools. For cohesin peak calling, MACS2 (Feng et al., 2012) callPeak was used. Adjacent and multiple Rad 21 peaks were annotated to 3 '-end of convergent genes. When multiple peaks were annotated to a 3'-end of convergent genes, a peak with maximum intensity at the converging intergenic sites was selected. 
Cells were spotted onto solid YES medium at 5-fold serial dilutions (in water) using a 48-pin replicator, and the plates were incubated at $30^{\circ} \mathrm{C}$ for 3 to 4 days.

\section{Protein extraction and Western blot analysis}

To check the expression level of Rad21 by SDS-PAGE gel analysis, whole-cell protein extracts were prepared either by a standard extraction protocol (Matsuo et al., 2006) or by a bead-beating method in which cells were resuspended with Laemmli sample buffer (4\% SDS, $40 \mathrm{mM}$ Tris-HCl, pH 6.8, 5\% glycerol, $5 \% \beta$-mercaptoethanol, $0.04 \%$ bromophenol blue) and lysed with glass beads.

\section{Microscopic analysis}

Prepared yeast colonies were spread on a coverglass-bottom dish (100350, SPL) and covered with a drop of soft agar. Images of whole yeast cells were captured by Z-stacking at a depth of $6 \mu \mathrm{m}$ and steps of $0.3 \mu \mathrm{m}$, using a 488-nm laser and a Nikon A1R plus confocal microscope fitted with a CFI plan Apochromat $\lambda$ DM 100x oil objective lens (1.45 numerical aperture, Nikon). Images were acquired at a resolution of 1024 x 1024 using a $3 \times$ digital zoom. GFP images were further evaluated with an Olympus IX-81 inverted fluorescence microscope.

\section{Real-time reverse transcrption PCR and library preparation for mRNA-sequencing}

Total RNA was extracted using the hot-phenol method (Schmitt et al., 1990) and treated with recombinant DnaseI (Ambion). For real-time reverse transcription PCR, either an oligo(dT) $)_{15}$ primer or a gene-specific primer were used with ImPromII Reverse Transcriptase (Promega). All primers used in RT-qPCR were listed in Supplemental Table 4.

For RNA-seq, DNase-treated mRNA was purified using an NEBNext ${ }^{\circledR}$ Poly(A) mRNA Magnetic Isolation Module (E7490S, NEB) and subjected to library preparation using a NEXTflex ${ }^{\text {TM }}$ Rapid Directional mRNA-Seq kit (5138-10, BIOO) according to the manufacturer's instructions. Each library was sequenced on a HiSeq2500 using the single-end method (50-bp reads). The sequenced reads were aligned to the S. pombe genome (ASM294v2) using the STAR aligner (Dobin et al., 2013). Strandspecific signal tracks of RNA-seq were generated by using bam2wig.py from RSeQC (Wang et al., 2012) with options --wigsum $=1,000,000,000$.

\section{Small RNA purification and library preparation for small RNA-sequencing}

Small RNAs preparation and small RNA-seq were performed as previously described (Seo et al., 2017). Briefly, small RNAs were purified and subjected to library construction using a NEXTFlex small RNAseq (5132-05, BIOO) according to the manufacturer's instructions. Each library was sequenced on a HiSeq2500 using the single-end method (50-bp reads). The small RNA-seq reads were further analyzed with the same method adopted in mRNA-sequencing analysis.

\section{Antibodies}


899 The following antibodies were used for the immunoblot or chromatin immunoprecipitation analyses: 900 anti- $\beta$-actin (sc47778, Santa Cruz), anti-FLAG (F1804, Sigma), anti-H3K9me3 (ab8898, Abcam), anti901 Rad21 (ab992, Abcam), anti-CTCF (07-729, Millipore), anti-V5 (MCA1360, Bio-Rad), and anti-CTD902 Ser2P (ab5095, Abcam).

903

\section{Data and Software Availability}

905 The RNA-seq, ChIP-seq, and Hi-C seq datasets have been deposited to the GEO under accession 906 number GSE85147. Correspondence and materials requests should be addressed to D.L. 907 (daeyoup@kaist.ac.kr). 


\section{Reference}

Akdemir KC, Chin L. 2015. HiCPlotter integrates genomic data with interaction matrices. Genome Biol 16. doi:10.1186/s13059-015-0767-1

Bähler J, Wu JQ, Longtine MS, Shah NG, McKenzie A, Steever AB, Wach A, Philippsen P, Pringle JR. 1998. Heterologous modules for efficient and versatile PCR-based gene targeting in Schizosaccharomyces pombe. Yeast 14:943-951. doi:10.1002/(SICI)10970061(199807)14:10<943::AID-YEA292>3.0.CO;2-Y

Bartkuhn M, Straub T, Herold M, Herrmann M, Rathke C, Saumweber H, Gilfillan GD, Becker PB, Renkawitz R. 2009. Active promoters and insulators are marked by the centrosomal protein 190. EMBO J 28:877-888. doi:10.1038/emboj.2009.34

Bernard P, Maure JF, Partridge JF, Genier S, Javerzat JP, Allshire RC. 2001. Requirement of heterochromatin for cohesion at centromeres. Science (80- ) 294:2539-2542. doi:10.1126/science. 1064027

Bernard P, Schmidt CK, Vaur S, Dheur S, Drogat J, Genier S, Ekwall K, Uhlmann F, Javerzat JP. 2008. Cell-cycle regulation of cohesin stability along fission yeast chromosomes. EMBO J 27:111-121. doi:10.1038/sj.emboj.7601955

Bhardwaj S, Schlackow M, Rabajdova M, Gullerova M. 2016. Transcription facilitates sister chromatid cohesion on chromosomal arms. Nucleic Acids Res 44:6676-6692. doi:10.1093/nar/gkw252

Bowen NJ, Jordan IK, Epstein JA, Wood V, Levin HL. 2003. Retrotransposons and their recognition of pol II promoters: A comprehensive survey of the transposable elements from the complete genome sequence of Schizosaccharomyces pombe. Genome Res 13:1984-1997. doi:10.1101/gr.1191603

Busslinger GA, Stocsits RR, Van Der Lelij P, Axelsson E, Tedeschi A, Galjart N, Peters JM. 2017. Cohesin is positioned in mammalian genomes by transcription, CTCF and Wapl. Nature 544:503507. doi:10.1038/nature22063

Callahan KP, Butler JS. 2010. TRAMP complex enhances RNA degradation by the nuclear exosome component Rrp6. J Biol Chem 285:3540-3547. doi:10.1074/jbc.M109.058396

Chan SL, Huppertz I, Yao C, Weng L, Moresco JJ, Yates JR, Ule J, Manley JL, Shi Y. 2014. CPSF30 and Wdr33 directly bind to AAUAAA in mammalian mRNA 3' processing. Genes Dev 28:2370 2380. doi:10.1101/gad.250993.114

Clerici M, Faini M, Muckenfuss LM, Aebersold R, Jinek M. 2018. Structural basis of AAUAAA polyadenylation signal recognition by the human CPSF complex. Nat Struct Mol Biol 25:135-138. doi:10.1038/s41594-017-0020-6

Colin J, Libri D, Porrua O. 2011. Cryptic Transcription and Early Termination in the Control of Gene Expression. Genet Res Int 2011:1-10. doi:10.4061/2011/653494

Costantino L, Hsieh THS, Lamothe R, Darzacq X, Koshland D. 2020. Cohesin residency determines chromatin loop patterns. Elife 9:1-31. doi:10.7554/eLife.59889

Crane E, Bian Q, McCord RP, Lajoie BR, Wheeler BS, Ralston EJ, Uzawa S, Dekker J, Meyer BJ. 2015. Condensin-driven remodelling of $\mathrm{X}$ chromosome topology during dosage compensation. Nature 523:240-244. doi:10.1038/nature14450

Dauban L, Montagne R, Thierry A, Lazar-Stefanita L, Bastié N, Gadal O, Cournac A, Koszul R, Beckouët F. 2020. Regulation of Cohesin-Mediated Chromosome Folding by Eco1 and Other Partners. Mol Cell 77:1279-1293.e4. doi:10.1016/j.molcel.2020.01.019

De Almeida SF, García-Sacristán A, Custódio N, Carmo-Fonseca M. 2010. A link between nuclear RNA 
surveillance, the human exosome and RNA polymerase II transcriptional termination. Nucleic Acids Res 38:8015-8026. doi:10.1093/nar/gkq703

Dixon JR, Selvaraj S, Yue F, Kim A, Li Y, Shen Y, Hu M, Liu JS, Ren B. 2012. Topological domains in mammalian genomes identified by analysis of chromatin interactions. Nature 485:376-380. doi:10.1038/nature11082

Dobin A, Davis CA, Schlesinger F, Drenkow J, Zaleski C, Jha S, Batut P, Chaisson M, Gingeras TR. 2013. STAR: Ultrafast universal RNA-seq aligner. Bioinformatics 29:15-21. doi:10.1093/bioinformatics/bts635

Dong F, Jiang J. 1998. Non-Rabl patterns of centromere and telomere distribution in the interphase nuclei of plant cells. Chromosom Res 6:551-558. doi:10.1023/A:1009280425125

Durand NC, Shamim MS, Machol I, Rao SSP, Huntley MH, Lander ES, Aiden EL. 2016. Juicer Provides a One-Click System for Analyzing Loop-Resolution Hi-C Experiments. Cell Syst 3:9598. doi:10.1016/j.cels.2016.07.002

Eaton JD, Francis L, Davidson L, West S. 2020. A unified allosteric/torpedo mechanism for transcriptional termination on human protein-coding genes. Genes Dev 34:132-145. doi:10.1101/gad.332833.119

Feng J, Liu T, Qin B, Zhang Y, Liu XS. 2012. Identifying ChIP-seq enrichment using MACS. Nat Protoc 7:1728-1740. doi:10.1038/nprot.2012.101

Fox MJ, Gao H, Smith-Kinnaman WR, Liu Y, Mosley AL. 2015. The Exosome Component Rrp6 Is Required for RNA Polymerase II Termination at Specific Targets of the Nrd1-Nab3 Pathway. PLoS Genet 11:1-26. doi:10.1371/journal.pgen.1004999

Fudenberg G, Imakaev M, Lu C, Goloborodko A, Abdennur N, Mirny LA. 2016. Formation of Chromosomal Domains by Loop Extrusion. Cell Rep 15:2038-2049. doi:10.1016/j.celrep.2016.04.085

Funabiki H, Hagan I, Uzawa S, Yanagida M. 1993. Cell cycle-dependent specific positioning and clustering of centromeres and telomeres in fission yeast. $J$ Cell Biol 121:961-976. doi:10.1083/jcb.121.5.961

Goloborodko A, Marko JF, Mirny LA. 2016. Chromosome Compaction by Active Loop Extrusion. Biophys J 110:2162-2168. doi:10.1016/j.bpj.2016.02.041

Graham AC, Kiss DL, Andrulis ED. 2009. Core exosome - Independent roles for Rrp6 in cell cycle progression. Mol Biol Cell 20:2242-2253. doi:10.1091/mbc.E08-08-0825

Gullerova M, Proudfoot NJ. 2008. Cohesin Complex Promotes Transcriptional Termination between Convergent Genes in S. pombe. Cell 132:983-995. doi:10.1016/j.cell.2008.02.040

Haarhuis JHI, van der Weide RH, Blomen VA, Yáñez-Cuna JO, Amendola M, van Ruiten MS, Krijger PHL, Teunissen H, Medema RH, van Steensel B, Brummelkamp TR, de Wit E, Rowland BD. 2017. The Cohesin Release Factor WAPL Restricts Chromatin Loop Extension. Cell 169:693707.e14. doi:10.1016/j.cell.2017.04.013

Hadjur S, Williams LM, Ryan NK, Cobb BS, Sexton T, Fraser P, Fisher AG, Merkenschlager M. 2009. Cohesins form chromosomal cis-interactions at the developmentally regulated IFNG locus. Nature 460:410-413. doi:10.1038/nature08079

Haering CH, Farcas AM, Arumugam P, Metson J, Nasmyth K. 2008. The cohesin ring concatenates sister DNA molecules. Nature 454:297-301. doi:10.1038/nature07098

Heinz S, Texari L, Hayes MGB, Urbanowski M, Chang MW, Givarkes N, Rialdi A, White KM, Albrecht RA, Pache L, Marazzi I, García-Sastre A, Shaw ML, Benner C. 2018. Transcription Elongation 
Can Affect Genome 3D Structure. Cell 174:1522-1536.e22. doi:10.1016/j.cell.2018.07.047

Hsieh THS, Weiner A, Lajoie B, Dekker J, Friedman N, Rando OJ. 2015. Mapping Nucleosome Resolution Chromosome Folding in Yeast by Micro-C. Cell 162:108-119. doi:10.1016/j.cell.2015.05.048

Izban MG, Luse DS. 1992. The RNA polymerase II ternary complex cleaves the nascent transcript in a $3^{\prime} \rightarrow 5^{\prime}$ direction in the presence of elongation factor SII. Genes Dev 6:1342-1356. doi:10.1101/gad.6.7.1342

Jin QW, Fuchs J, Loidl J. 2000. Centromere clustering is a major determinant of yeast interphase nuclear organization. J Cell Sci 113:1903-1912.

Kim KD, Tanizawa H, Iwasaki O, Noma KI. 2016. Transcription factors mediate condensin recruitment and global chromosomal organization in fission yeast. Nat Genet 48:1242-1252. doi:10.1038/ng.3647

Kim SK, Lee H, Han K, Kim SC, Choi Y, Park SW, Bak G, Lee Y, Choi JK, Kim TK, Han YM, Lee D. 2014. SET7/9 methylation of the pluripotency factor LIN28A is a nucleolar localization mechanism that blocks let-7 biogenesis in human ESCs. Cell Stem Cell 15:735-749. doi:10.1016/j.stem.2014.10.016

Kinoshita N, Goebl M, Yanagida M. 1991. The fission yeast dis3+ gene encodes a 110-kDa essential protein implicated in mitotic control. Mol Cell Biol 11:5839-5847. doi:10.1128/mcb.11.12.5839

Kueng S, Hegemann B, Peters BH, Lipp JJ, Schleiffer A, Mechtler K, Peters JM. 2006. Wapl Controls the Dynamic Association of Cohesin with Chromatin. Cell 127:955-967. doi:10.1016/j.cell.2006.09.040

Langmead B, Salzberg SL. 2012. Fast gapped-read alignment with Bowtie 2. Nat Methods 9:357-359. doi:10.1038/nmeth.1923

Larochelle M, Robert MA, Hébert JN, Liu X, Matteau D, Rodrigue S, Tian B, Jacques PÉ, Bachand F. 2018. Common mechanism of transcription termination at coding and noncoding RNA genes in fission yeast. Nat Commun 9. doi:10.1038/s41467-018-06546-x

Lemay JF, Larochelle M, Marguerat S, Atkinson S, Bähler J, Bachand F. 2014. The RNA exosome promotes transcription termination of backtracked RNA polymerase II. Nat Struct Mol Biol 21:919-926. doi:10.1038/nsmb.2893

Lengronne A, Katou Y, Mori S, Yokabayashi S, Kelly GP, Ito T, Watanabe Y, Shirahige K, Uhlmann F. 2004. Cohesin relocation from sites of chromosomal loading to places of convergent transcription. Nature 430:573-578. doi:10.1038/nature02742

Lieberman-Aiden E, Van Berkum NL, Williams L, Imakaev M, Ragoczy T, Telling A, Amit I, Lajoie BR, Sabo PJ, Dorschner MO, Sandstrom R, Bernstein B, Bender MA, Groudine M, Gnirke A, Stamatoyannopoulos J, Mirny LA, Lander ES, Dekker J. 2009. Comprehensive mapping of longrange interactions reveals folding principles of the human genome. Science (80-) 326:289-293. doi:10.1126/science. 1181369

Liu Q, Greimann JC, Lima CD. 2006. Reconstitution, Activities, and Structure of the Eukaryotic RNA Exosome. Cell 127:1223-1237. doi:10.1016/j.cell.2006.10.037

Losada A, Hirano M, Hirano T. 1998. Identification of Xenopus SMC protein complexes required for sister chromatid cohesion. Genes Dev 12:1986-1997. doi:10.1101/gad.12.13.1986

Matsuo Y, Asakawa K, Toda T, Katayama S. 2006. A rapid method for protein extraction from fission yeast. Biosci Biotechnol Biochem 70:1992-1994. doi:10.1271/bbb.60087

Matthews NE, White R. 2019. Chromatin Architecture in the Fly: Living without CTCF/Cohesin Loop 
Extrusion?: Alternating Chromatin States Provide a Basis for Domain Architecture in Drosophila. BioEssays 41. doi:10.1002/bies.201900048

Minajigi A, Froberg JE, Wei C, Sunwoo H, Kesner B, Colognori D, Lessing D, Payer B, Boukhali M, Haas W, Lee JT. 2015. A comprehensive Xist interactome reveals cohesin repulsion and an RNAdirected chromosome conformation. Science (80- ) 349:1DUIMMY. doi:10.1126/science.aab2276

Mizuguchi T, Barrowman J, Grewal SIS. 2015. Chromosome domain architecture and dynamic organization of the fission yeast genome. FEBS Lett 589:2975-2986. doi:10.1016/j.febslet.2015.06.008

Mizuguchi T, Fudenberg G, Mehta S, Belton JM, Taneja N, Folco HD, FitzGerald P, Dekker J, Mirny L, Barrowman J, Grewal SIS. 2014. Cohesin-dependent globules and heterochromatin shape 3D genome architecture in S. pombe. Nature 516:432-435. doi:10.1038/nature13833

Mukarami H, Goto DB, Toda T, Chen ES, Grewal SI, Martienssen RA, Yanagida M. 2007. Ribonuclease activity of Dis3 is required for mitotic progression and provides a possible link between heterochromatin and kinetochore function. PLoS One 2. doi:10.1371/journal.pone.0000317

Murayama Y, Samora CP, Kurokawa Y, Iwasaki H, Uhlmann F. 2018. Establishment of DNA-DNA Interactions by the Cohesin Ring. Cell 172:465-477.e15. doi:10.1016/j.cell.2017.12.021

Nabeshima K, Nakagawa T, Straight AF, Murray A, Chikashige Y, Yamashita YM, Hiraoka Y, Yanagida M. 1998. Dynamics of centromeres during metaphase-anaphase transition in fission yeast: Dis1 is implicated in force balance in metaphase bipolar spindle. Mol Biol Cell 9:3211-3225. doi:10.1091/mbc.9.11.3211

Nonaka N, Kitajima T, Yokobayashi S, Xiao G, Yamamoto M, Grewal SIS, Watanabe Y. 2002. Recruitment of cohesin to heterochromatic regions by Swi6/HP1 in fission yeast. Nat Cell Biol 4:89-93. doi:10.1038/ncb739

Nora EP, Goloborodko A, Valton AL, Gibcus JH, Uebersohn A, Abdennur N, Dekker J, Mirny LA, Bruneau BG. 2017. Targeted Degradation of CTCF Decouples Local Insulation of Chromosome Domains from Genomic Compartmentalization. Cell 169:930-944.e22. doi:10.1016/j.cell.2017.05.004

Nora EP, Lajoie BR, Schulz EG, Giorgetti L, Okamoto I, Servant N, Piolot T, Van Berkum NL, Meisig J, Sedat J, Gribnau J, Barillot E, Blüthgen N, Dekker J, Heard E. 2012. Spatial partitioning of the regulatory landscape of the X-inactivation centre. Nature 485:381-385. doi:10.1038/nature11049

O’Sullivan JM, Tan-Wong SM, Morillon A, Lee B, Coles J, Mellor J, Proudfoot NJ. 2004. Gene loops juxtapose promoters and terminators in yeast. Nat Genet 36:1014-1018. doi:10.1038/ng1411

Ohkura H, Adachi Y, Kinoshita N, Niwa O, Toda T, Yanagida M. 1988. Cold-sensitive and caffeinesupersensitive mutants of the Schizosaccharomyces pombe dis genes implicated in sister chromatid separation during mitosis. EMBO $J$ 7:1465-1473. doi:10.1002/j.14602075.1988.tb02964.x

Ohkura H, Kinoshita N, Miyatani S, Toda T, Yanagida M. 1989. The fission yeast dis2+ gene required for chromosome disjoining encodes one of two putative type 1 protein phosphatases. Cell 57:9971007. doi:10.1016/0092-8674(89)90338-3

Onn I, Heidinger-Pauli JM, Guacci V, Ünal E, Koshland DE. 2008. Sister chromatid cohesion: A simple concept with a complex reality. Annu Rev Cell Dev Biol 24:105-129. doi:10.1146/annurev.cellbio.24.110707.175350

Paldi F, Alver B, Robertson D, Schalbetter SA, Kerr A, Kelly DA, Baxter J, Neale MJ, Marston AL. 2020. Convergent genes shape budding yeast pericentromeres. Nature 582:119-123. doi:10.1038/s41586-020-2244-6 
Pan H, Jin M, Ghadiyaram A, Kaur P, Miller HE, Ta HM, Liu M, Fan Y, Mahn C, Gorthi A, You C, Piehler J, Riehn R, Bishop AJR, Tao YJ, Wang H. 2021. Cohesin SA1 and SA2 are RNA binding proteins that localize to RNA containing regions on DNA. Nucleic Acids Res 48:5639-5655. doi:10.1093/NAR/GKAA284

Parelho V, Hadjur S, Spivakov M, Leleu M, Sauer S, Gregson HC, Jarmuz A, Canzonetta C, Webster Z, Nesterova T, Cobb BS, Yokomori K, Dillon N, Aragon L, Fisher AG, Merkenschlager M. 2008. Cohesins Functionally Associate with CTCF on Mammalian Chromosome Arms. Cell 132:422433. doi:10.1016/j.cell.2008.01.011

Parsa JY, Boudoukha S, Burke J, Homer C, Madhani HD. 2018. Polymerase pausing induced by sequence-specific RNA-binding protein drives heterochromatin assembly. Genes Dev 32:953-964. doi:10.1101/gad.310136.117

Peters JM, Tedeschi A, Schmitz J. 2008. The cohesin complex and its roles in chromosome biology. Genes Dev 22:3089-3114. doi:10.1101/gad.1724308

Pokholok DK, Harbison CT, Levine S, Cole M, Hannett NM, Tong IL, Bell GW, Walker K, Rolfe PA, Herbolsheimer E, Zeitlinger J, Lewitter F, Gifford DK, Young RA. 2005. Genome-wide map of nucleosome acetylation and methylation in yeast. Cell 122:517-527. doi:10.1016/j.cell.2005.06.026

Ramírez F, Bhardwaj V, Arrigoni L, Lam KC, Grüning BA, Villaveces J, Habermann B, Akhtar A, Manke T. 2018. High-resolution TADs reveal DNA sequences underlying genome organization in flies. Nat Commun 9. doi:10.1038/s41467-017-02525-w

Ramírez F, Ryan DP, Grüning B, Bhardwaj V, Kilpert F, Richter AS, Heyne S, Dündar F, Manke T. 2016. deepTools2: a next generation web server for deep-sequencing data analysis. Nucleic Acids Res 44:160-165. doi:10.1093/nar/gkw257

Rao SSP, Huntley MH, Durand NC, Stamenova EK, Bochkov ID, Robinson JT, Sanborn AL, Machol I, Omer AD, Lander ES, Aiden EL. 2014. A 3D map of the human genome at kilobase resolution reveals principles of chromatin looping. Cell 159:1665-1680. doi:10.1016/j.cell.2014.11.021

Reines D. 1992. Elongation factor-dependent transcript shortening by template-engaged RNA polymerase II. $J$ Biol Chem 267:3795-3800. doi:10.1016/s0021-9258(19)50596-8

Reyes-Turcu FE, Zhang K, Zofall M, Chen E, Grewal SIS. 2010. Defects in RNA quality control factors reveal RNAi-independent nucleation of heterochromatin. Nat Struct Mol Biol 18:1132-1138. doi: $10.1038 / \mathrm{nsmb} .2122$

Rivosecchi J, Jost D, Vachez L, Gautier FD, Bernard P, Vanoosthuyse V. 2021. RNA polymerase backtracking results in the accumulation of fission yeast condensin at active genes. Life Sci alliance 4. doi:10.26508/1sa.202101046

Rowley MJ, Corces VG. 2016. The three-dimensional genome: Principles and roles of long-distance interactions. Curr Opin Cell Biol 40:8-14. doi:10.1016/j.ceb.2016.01.009

Rowley MJ, Lyu X, Rana V, Ando-Kuri M, Karns R, Bosco G, Corces VG. 2019. Condensin II Counteracts Cohesin and RNA Polymerase II in the Establishment of 3D Chromatin Organization. Cell Rep 26:2890-2903.e3. doi:10.1016/j.celrep.2019.01.116

Rowley MJ, Nichols MH, Lyu X, Ando-Kuri M, Rivera ISM, Hermetz K, Wang P, Ruan Y, Corces VG. 2017. Evolutionarily Conserved Principles Predict 3D Chromatin Organization. Mol Cell 67:837852.e7. doi:10.1016/j.molcel.2017.07.022

Rubio ED, Reiss DJ, Welcsh PL, Disteche CM, Filippova GN, Baliga NS, Aebersold R, Ranish JA, Krumm A. 2008. CTCF physically links cohesin to chromatin. Proc Natl Acad Sci US A 105:8309-8314. doi:10.1073/pnas.0801273105 
Sanborn AL, Rao SSP, Huang SC, Durand NC, Huntley MH, Jewett AI, Bochkov ID, Chinnappan D, Cutkosky A, Li J, Geeting KP, Gnirke A, Melnikov A, McKenna D, Stamenova EK, Lander ES, Aiden EL. 2015. Chromatin extrusion explains key features of loop and domain formation in wildtype and engineered genomes. Proc Natl Acad Sci $U$ S A 112:E6456-E6465. doi:10.1073/pnas.1518552112

Schmidt CK, Brookes N, Uhlmann F. 2009. Conserved features of cohesin binding along fission yeast chromosomes. Genome Biol 10. doi:10.1186/gb-2009-10-5-r52

Schmitt ME, Brown TA, Trumpower BL. 1990. A rapid and simple method for preparation of RNA from Saccharomyces cerevisiae. Nucleic Acids Res 18:3091-3092. doi:10.1093/nar/18.10.3091

Schönemann L, Kühn U, Martin G, Schäfer P, Gruber AR, Keller W, Zavolan M, Wahle E. 2014. Reconstitution of CPSF active in polyadenylation: Recognition of the polyadenylation signal by WDR33. Genes Dev 28:2381-2393. doi:10.1101/gad.250985.114

Schwarzer W, Abdennur N, Goloborodko A, Pekowska A, Fudenberg G, Loe-Mie Y, Fonseca NA, Huber W, Haering CH, Mirny L, Spitz F. 2017. Two independent modes of chromatin organization revealed by cohesin removal. Nature 551:51-56. doi:10.1038/nature24281

Seo HD, Choi Y, Kim M, Kang K, Urano T, Lee D. 2017. The 19S proteasome is directly involved in the regulation of heterochromatin spreading in fission yeast. $J$ Biol Chem. doi:10.1074/jbc.M117.790824

Servant N, Varoquaux N, Lajoie BR, Viara E, Chen CJ, Vert JP, Heard E, Dekker J, Barillot E. 2015. HiC-Pro: An optimized and flexible pipeline for Hi-C data processing. Genome Biol 16. doi:10.1186/s13059-015-0831-x

Sheridan RM, Fong N, D’Alessandro A, Bentley DL. 2019. Widespread Backtracking by RNA Pol II Is a Major Effector of Gene Activation, 5' Pause Release, Termination, and Transcription Elongation Rate. Mol Cell 73:107-118.e4. doi:10.1016/j.molcel.2018.10.031

Shobuike T, Tatebayashi K, Tani T, Sugano S, Ikeda H. 2001. The dhp1+ gene, encoding a putative nuclear $5^{\prime} \rightarrow 3^{\prime}$ exoribonuclease, is required for proper chromosome segregation in fission yeast. Nucleic Acids Res 29:1326-1333. doi:10.1093/nar/29.6.1326

Sigurdsson S, Dirac-Svejstrup AB, Svejstrup JQ. 2010. Evidence that Transcript Cleavage Is Essential for RNA Polymerase II Transcription and Cell Viability. Mol Cell 38:202-210. doi:10.1016/j.molcel.2010.02.026

Strahl-Bolsinger S, Hecht A, Luo K, Grunstein M. 1997. SIR2 and SIR4 interactions differ in core and extended telomeric heterochromatin in yeast. Genes Dev 11:83-93. doi:10.1101/gad.11.1.83

Szankasi P, Smith GR. 1996. Requirement of S. pombe exonuclease II, a homologue of S. cerevisiae Sep1, for normal mitotic growth and viability. Curr Genet 30:284-293. doi:10.1007/s002940050134

Tan-Wong SM, Zaugg JB, Camblong J, Xu Z, Zhang DW, Mischo HE, Ansari AZ, Luscombe NM, Steinmetz LM, Proudfoot NJ. 2012. Gene loops enhance transcriptional directionality. Science (80- ) 338:671-675. doi:10.1126/science. 1224350

Tanizawa H, Kim KD, Iwasaki O, Noma KI. 2017. Architectural alterations of the fission yeast genome during the cell cycle. Nat Struct Mol Biol 24:965-976. doi:10.1038/nsmb.3482

Tatebayashi K, Kato JI, Ikeda H. 1998. Isolation of a Schizosaccharomyces pombe rad21(ts) mutant that is aberrant in chromosome segregation, microtubule function, DNA repair and sensitive to hydroxyurea: Possible involvement of $\operatorname{Rad} 21$ in ubiquitin-mediated proteolysis. Genetics 148:4957. 
Tóth A, Ciosk R, Uhlmann F, Galova M, Schleiffer A, Nasmyth K. 1999. Yeast cohesin complex requires a conserved protein, Ecolp(Ctf7), to establish cohesion between sister chromatids during DNA replication. Genes Dev 13:320-333. doi:10.1101/gad.13.3.320

Van Bortle K, Nichols MH, Li L, Ong CT, Takenaka N, Qin ZS, Corces VG. 2014. Insulator function and topological domain border strength scale with architectural protein occupancy. Genome Biol 15:R82. doi:10.1186/gb-2014-15-5-r82

Vara C, Paytuví-Gallart A, Cuartero Y, Le Dily F, Garcia F, Salvà-Castro J, Gómez-H L, Julià E, Moutinho C, Aiese Cigliano R, Sanseverino W, Fornas O, Pendás AM, Heyn H, Waters PD, MartiRenom MA, Ruiz-Herrera A. 2019. Three-Dimensional Genomic Structure and Cohesin Occupancy Correlate with Transcriptional Activity during Spermatogenesis. Cell Rep 28:352367.e9. doi:10.1016/j.celrep.2019.06.037

Vasiljeva L, Buratowski S. 2006. Nrd1 interacts with the nuclear exosome for 3' processing of RNA polymerase II transcripts. Mol Cell 21:239-248. doi:10.1016/j.molcel.2005.11.028

Vernì F, Gandhi R, Goldberg ML, Gatti M. 2000. Genetic and molecular analysis of wings apart-like (wapl), a gene controlling heterochromatin organization in Drosophila melanogaster. Genetics 154:1693-1710.

Wang L, Wang S, Li W. 2012. RSeQC: Quality control of RNA-seq experiments. Bioinformatics 28:2184-2185. doi:10.1093/bioinformatics/bts356

Wang S-W, Asakawa K, Win TZ, Toda T, Norbury CJ. 2005. Inactivation of the Pre-mRNA Cleavage and Polyadenylation Factor Pfs2 in Fission Yeast Causes Lethal Cell Cycle Defects. Mol Cell Biol 25:2288-2296. doi:10.1128/mcb.25.6.2288-2296.2005

Wendt KS, Yoshida K, Itoh T, Bando M, Koch B, Schirghuber E, Tsutsumi S, Nagae G, Ishihara K, Mishiro T, Yahata K, Imamoto F, Aburatani H, Nakao M, Imamoto N, Maeshima K, Shirahige K, Peters JM. 2008. Cohesin mediates transcriptional insulation by CCCTC-binding factor. Nature 451:796-801. doi:10.1038/nature06634

Win TZ, Draper S, Read RL, Pearce J, Norbury CJ, Wang S-W. 2006. Requirement of Fission Yeast Cid14 in Polyadenylation of rRNAs. Mol Cell Biol 26:1710-1721. doi:10.1128/mcb.26.5.17101721.2006

Wittmann S, Renner M, Watts BR, Adams O, Huseyin M, Baejen C, El Omari K, Kilchert C, Heo DH, Kecman T, Cramer P, Grimes JM, Vasiljeva L. 2017. The conserved protein Seb1 drives transcription termination by binding RNA polymerase II and nascent RNA. Nat Commun 8. doi:10.1038/ncomms14861

Wutz G, Várnai C, Nagasaka K, Cisneros DA, Stocsits RR, Tang W, Schoenfelder S, Jessberger G, Muhar M, Hossain MJ, Walther N, Koch B, Kueblbeck M, Ellenberg J, Zuber J, Fraser P, Peters J. 2017. Topologically associating domains and chromatin loops depend on cohesin and are regulated by CTCF, WAPL, and PDS5 proteins. EMBO $J$ 36:3573-3599. doi:10.15252/embj.201798004

Yamamoto A, Hiraoka Y. 2003. Monopolar spindle attachment of sister chromatids is ensured by two distinct mechanisms at the first meiotic division in fission yeast. EMBO $J$ 22:2284-2296. doi:10.1093/emboj/cdg222

Yamanaka S, Mehta S, Reyes-Turcu FE, Zhuang F, Fuchs RT, Rong Y, Robb GB, Grewal SIS. 2013. RNAi triggered by specialized machinery silences developmental genes and retrotransposons. Nature 493:557-560. doi:10.1038/nature11716

Yang T, Zhang F, Yardımci GG, Song F, Hardison RC, Noble WS, Yue F, Li Q. 2017. HiCRep: assessing the reproducibility of Hi-C data using a stratum-adjusted correlation coefficient. Genome Res 
Zhan Y, Mariani L, Barozzi I, Schulz EG, Blüthgen N, Stadler M, Tiana G, Giorgetti L. 2017. Reciprocal insulation analysis of Hi-C data shows that TADs represent a functionally but not structurally privileged scale in the hierarchical folding of chromosomes. Genome Res 27:479-490. doi:10.1101/gr.212803.116

Zhao N, Sebastiano V, Moshkina N, Mena N, Hultquist J, Jimenez-Morales D, Ma Y, Rialdi A, Albrecht R, Fenouil R, Sánchez-Aparicio MT, Ayllon J, Ravisankar S, Haddad B, Ho JSY, Low D, Jin J, Yurchenko V, Prinjha RK, Tarakhovsky A, Squatrito M, Pinto D, Allette K, Byun M, Smith ML, Sebra R, Guccione E, Tumpey T, Krogan N, Greenbaum B, van Bakel H, García-Sastre A, Marazzi I. 2018. Influenza virus infection causes global RNAPII termination defects. Nat Struct Mol Biol 25:885-893. doi:10.1038/s41594-018-0124-7

Zilio N, Wehrkamp-Richter S, Boddy MN. 2012. A new versatile system for rapid control of gene expression in the fission yeast Schizosaccharomyces pombe. Yeast 29:425-434. 


\section{Main Figure 1}

A

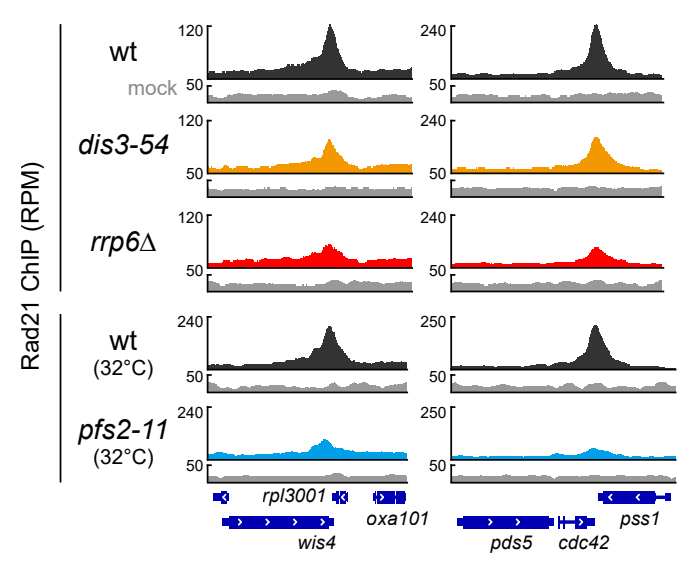

B

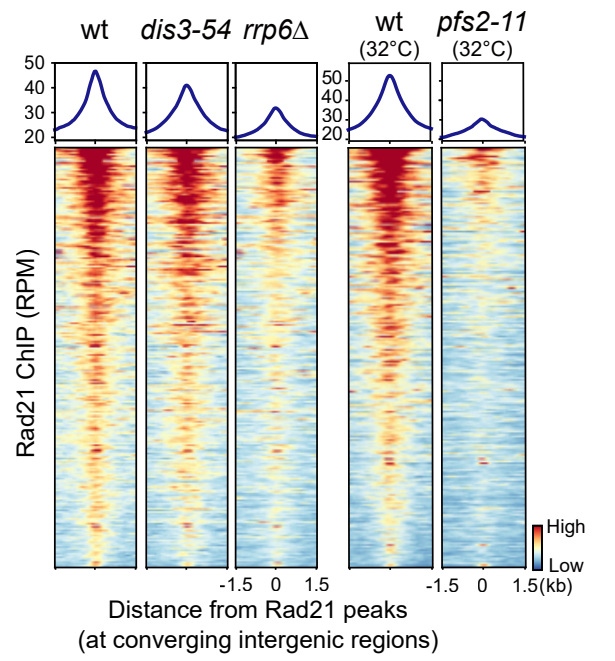

C

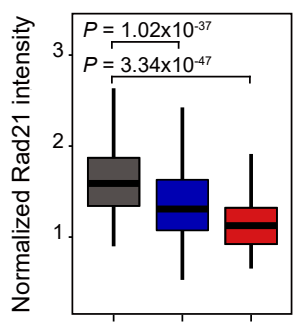

wt dis3-54 rrp6 $\Delta$

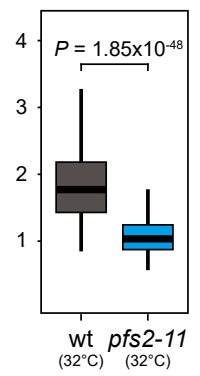

D

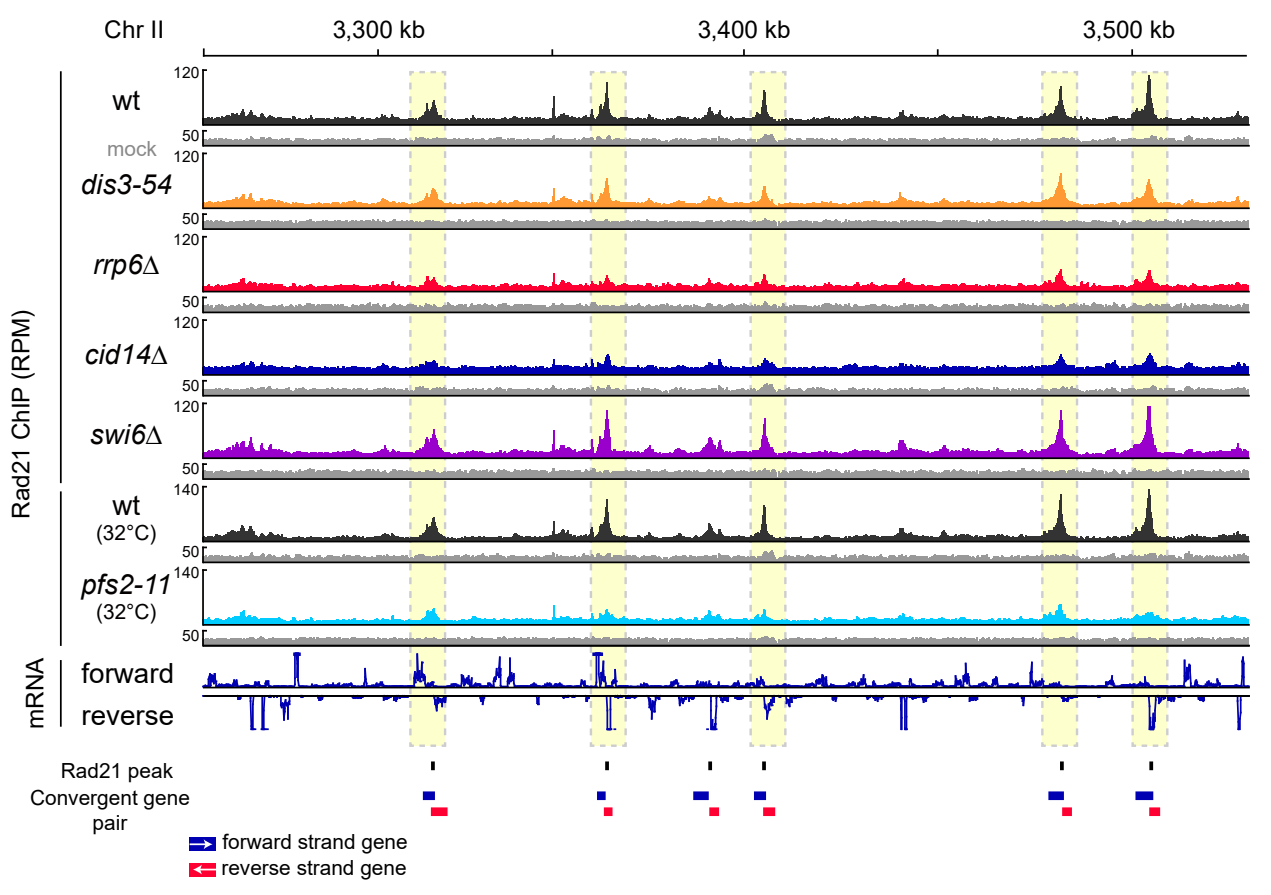




\section{Main Figure 2}

A
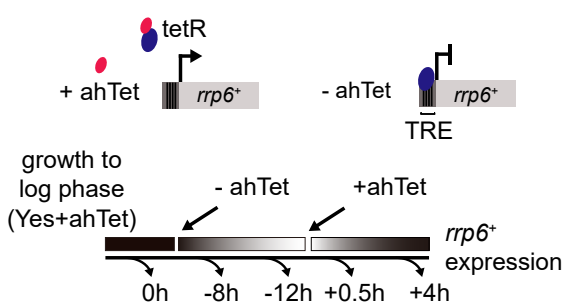

B

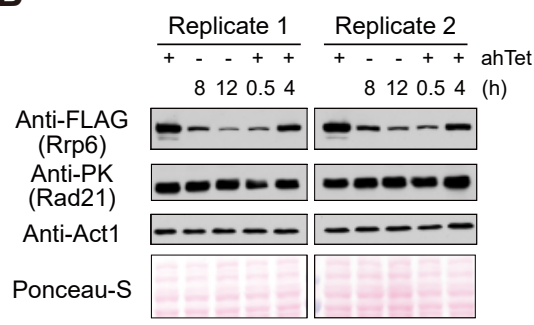

F

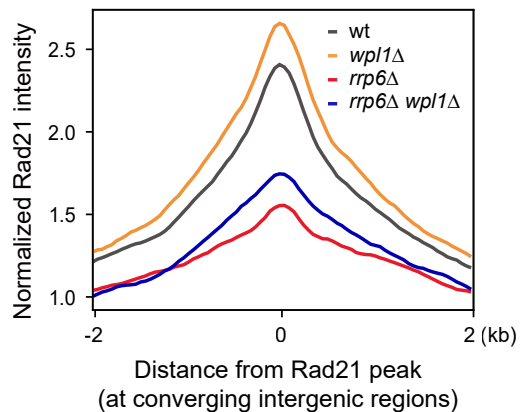

C

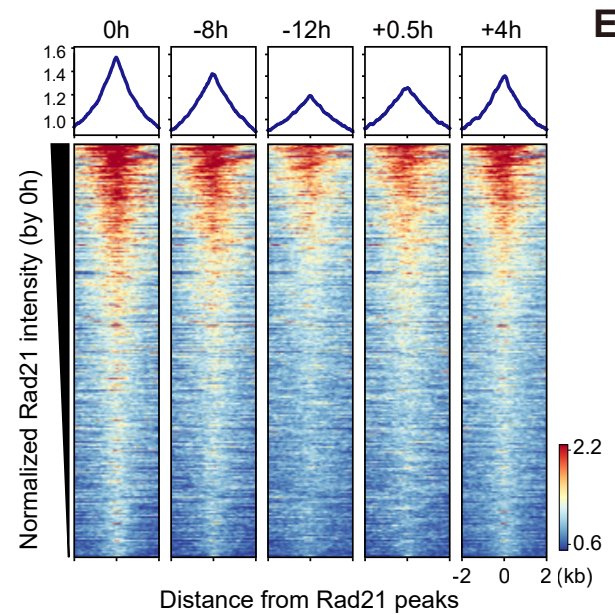

D (at converging intergenic regions)

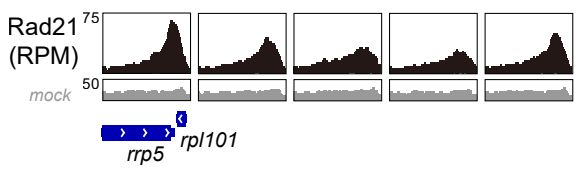

G

H

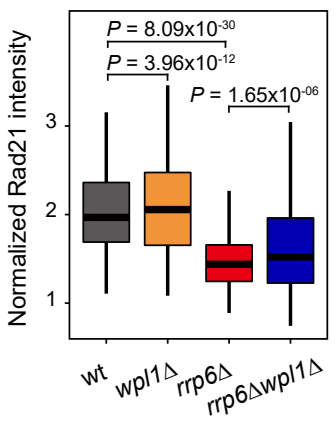

E
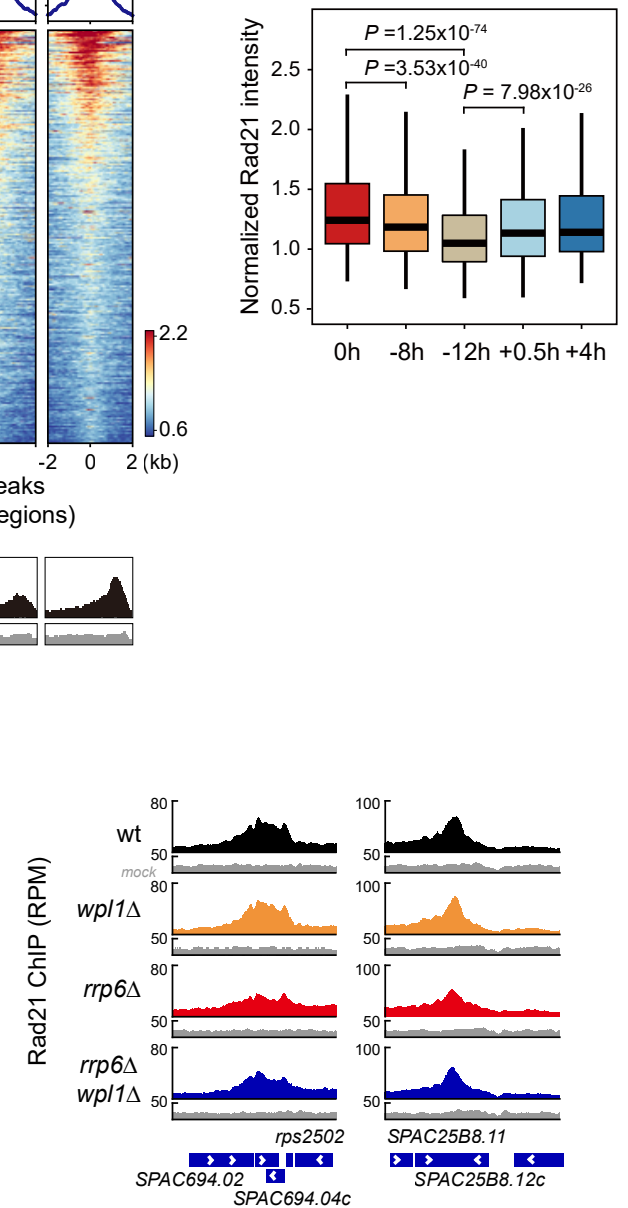


\section{Main Figure 3}

A
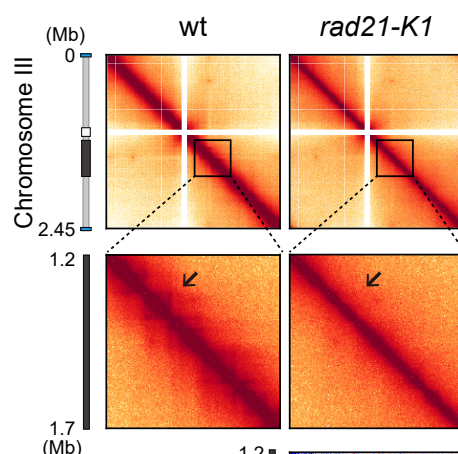

$$
\text { (Mb) }
$$

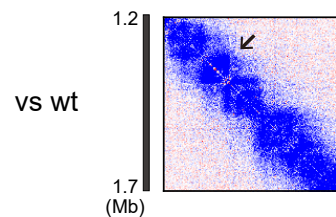

C

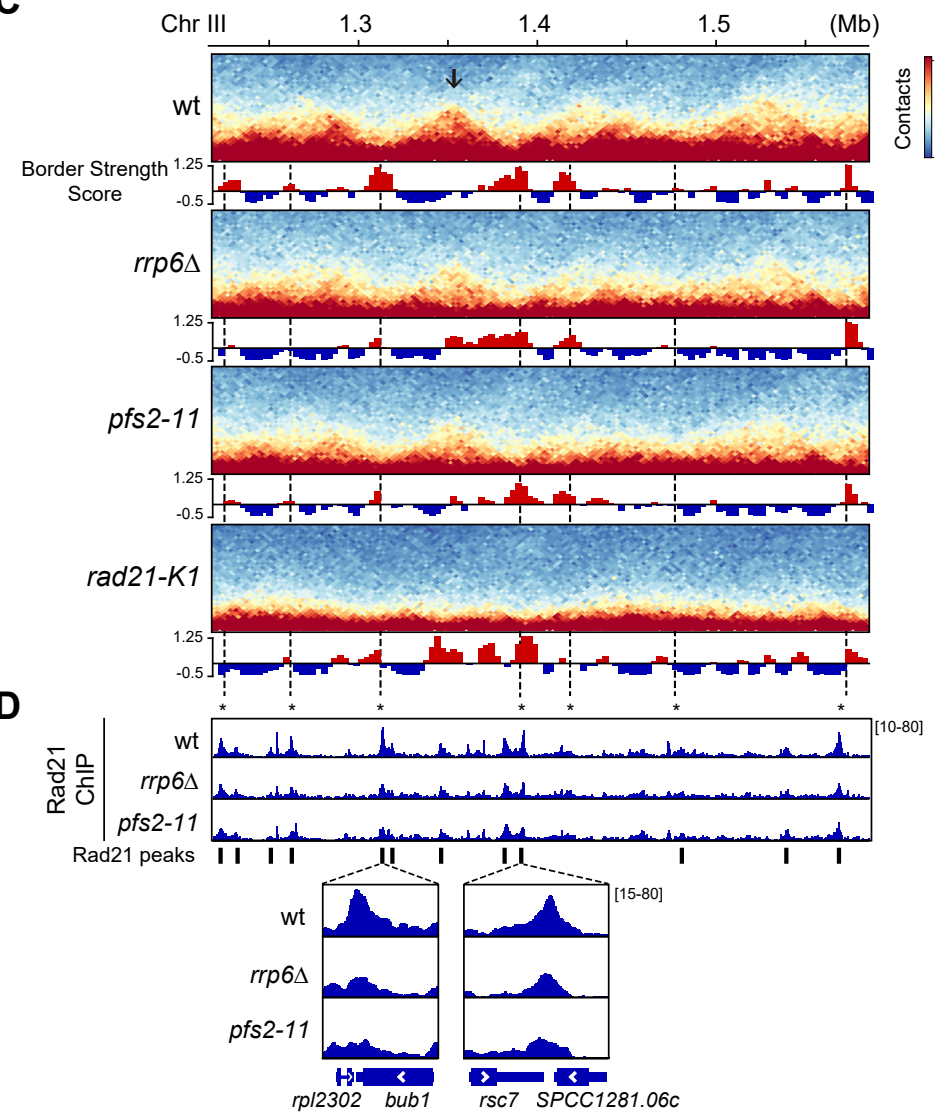

B
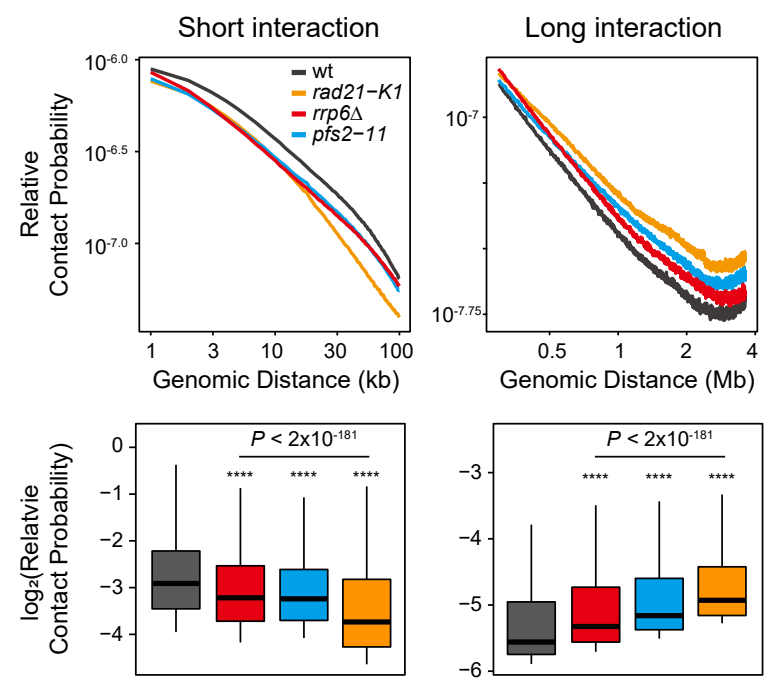

E

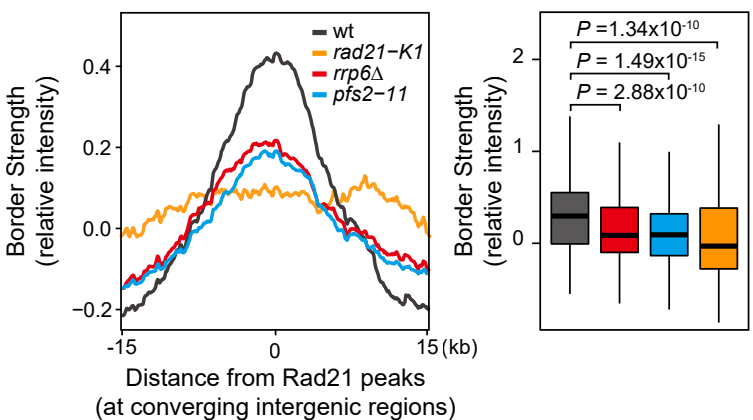

F

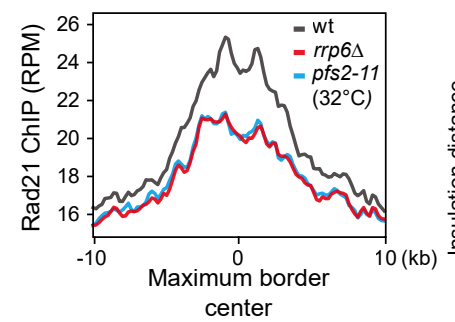

G

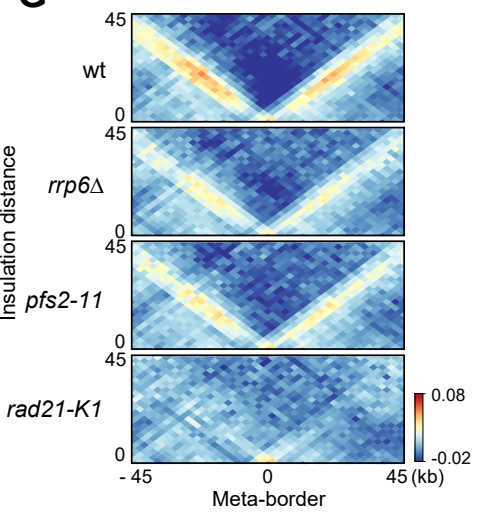




\section{Main Figure 4}

A

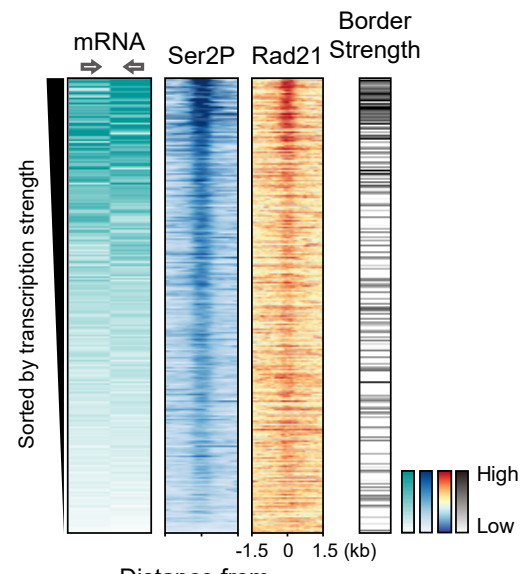

Distance from

converging intergenic center

D

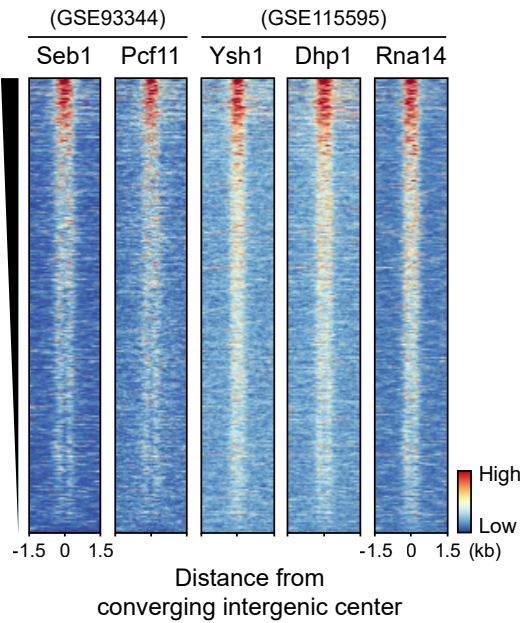

B

Symmetric expression

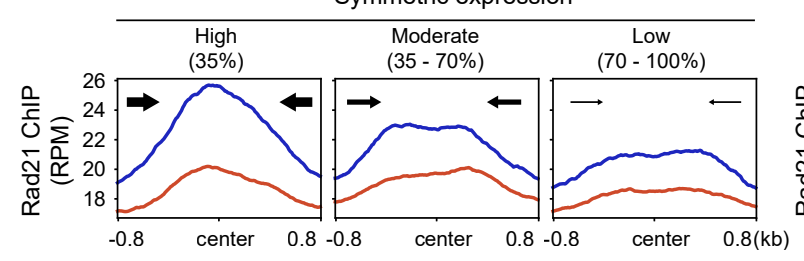

C
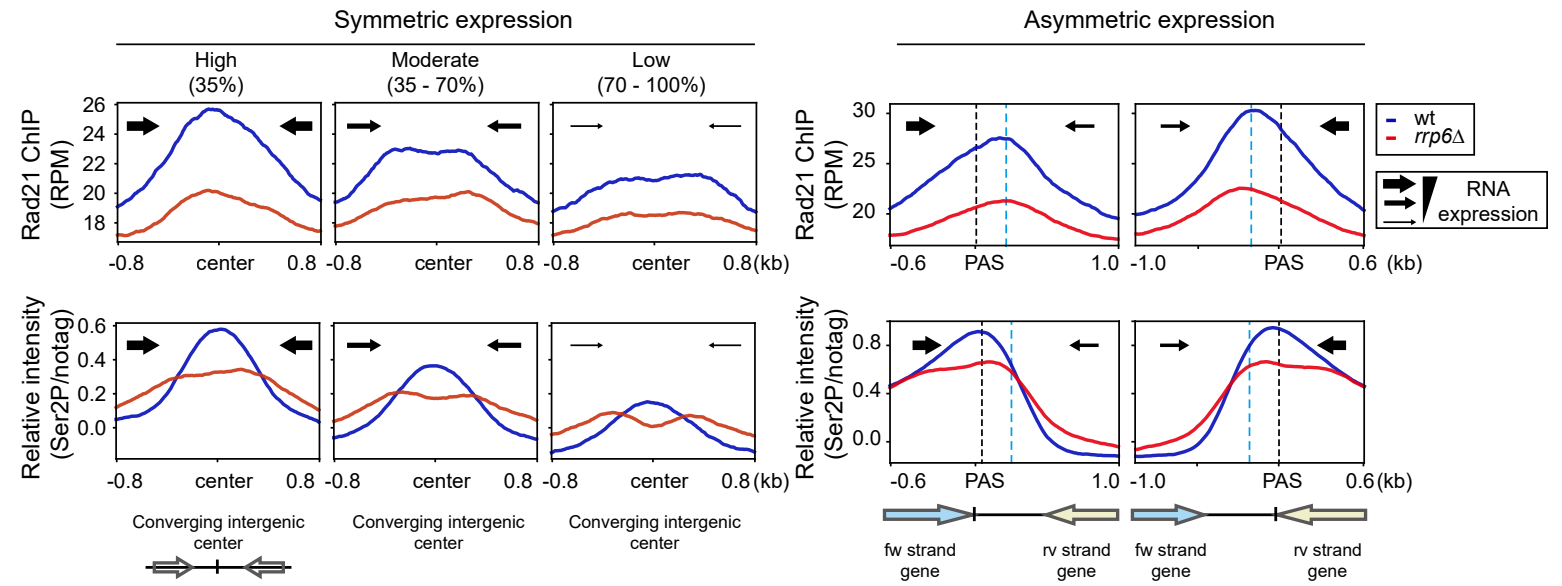

E

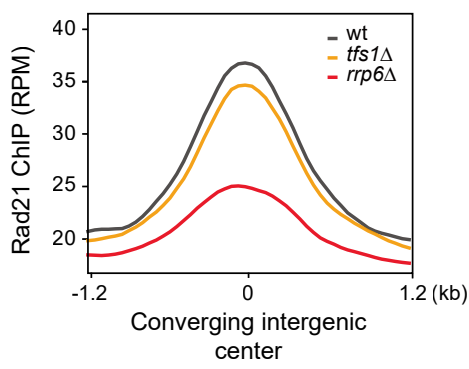

F

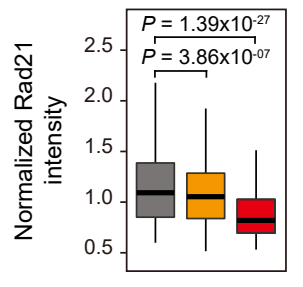

G

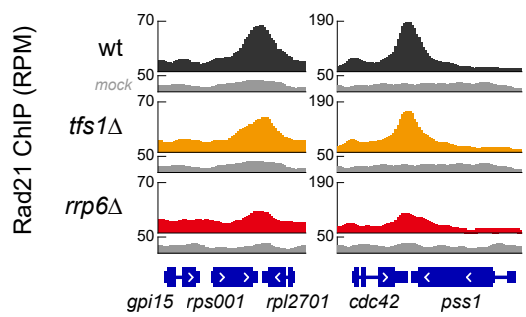




\section{Main Figure 5}

A

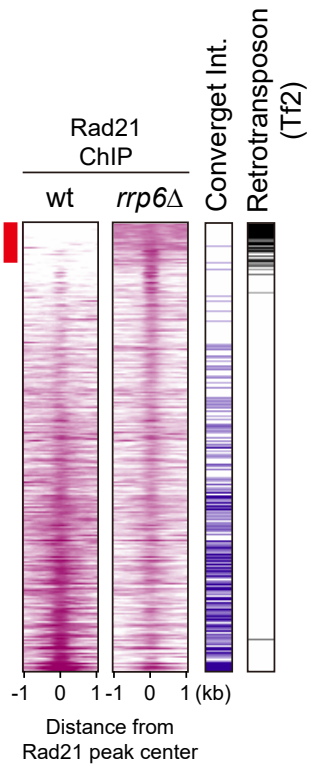

B

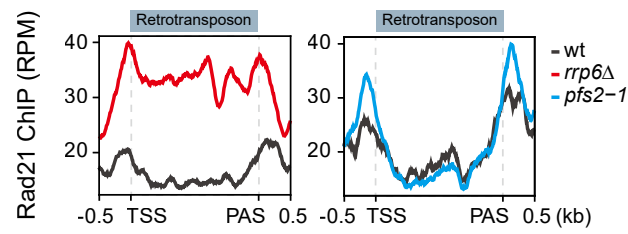

C

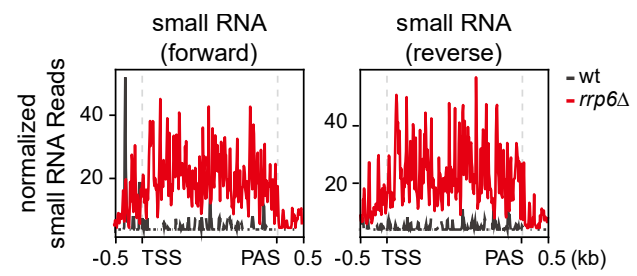

D

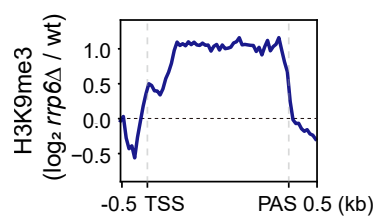

F
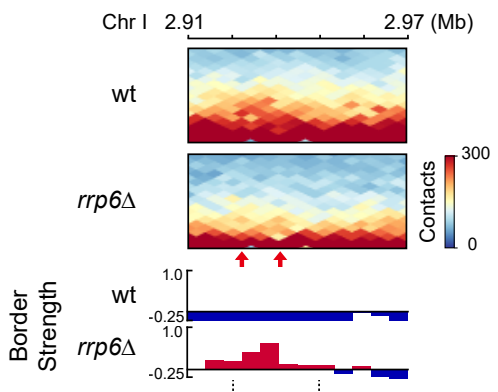

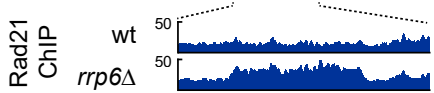

$\begin{array}{cc}\mathrm{H} 3 \mathrm{~K} 9 \mathrm{me} 3 & { }^{0.8} \\ \left(\log _{2} r \mathrm{rp} 6 \Delta / \mathrm{wt}\right) & -0.8 \mathrm{~m} / \mathrm{m}\end{array}$

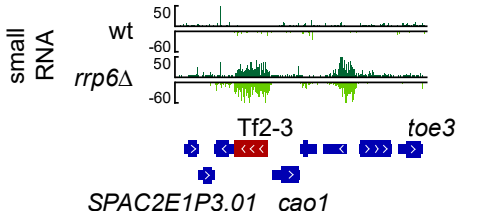

E

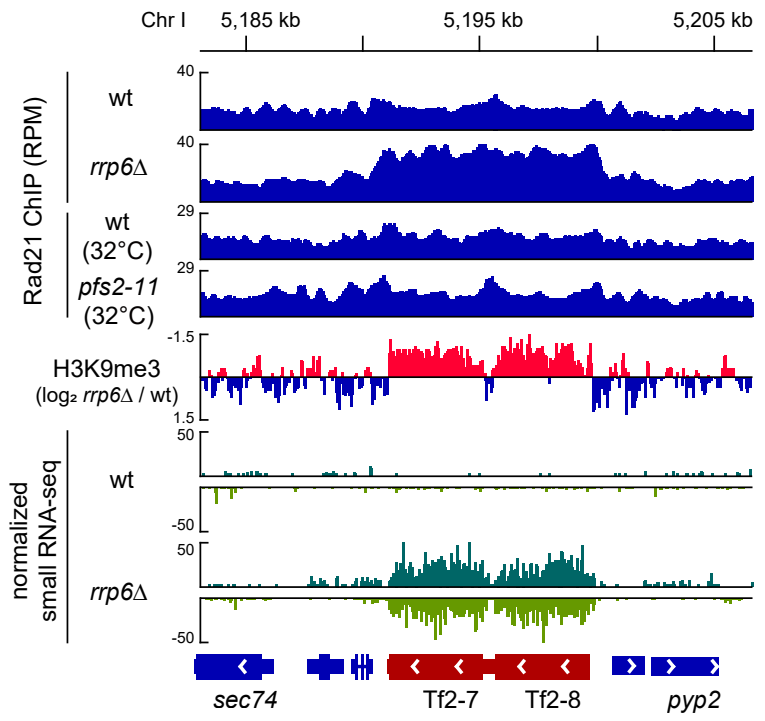

G

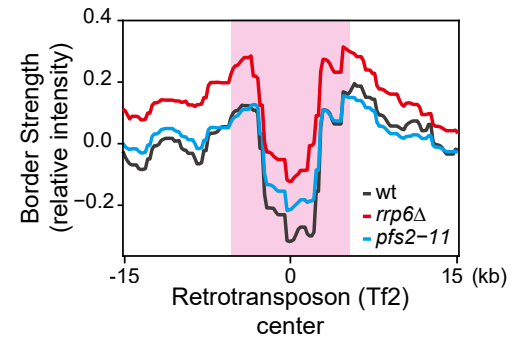

H

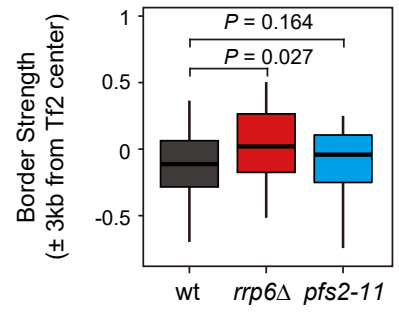




\section{Main Figure 6}

A

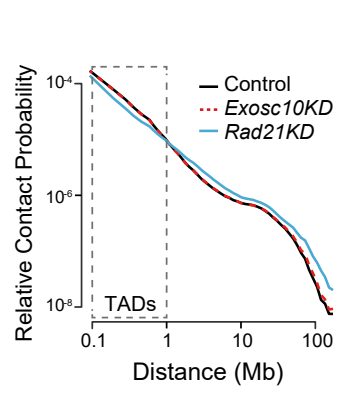

B

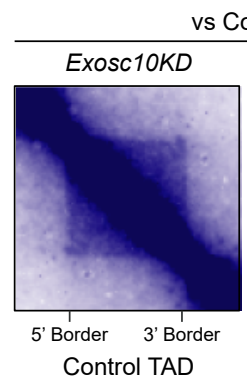

C

D
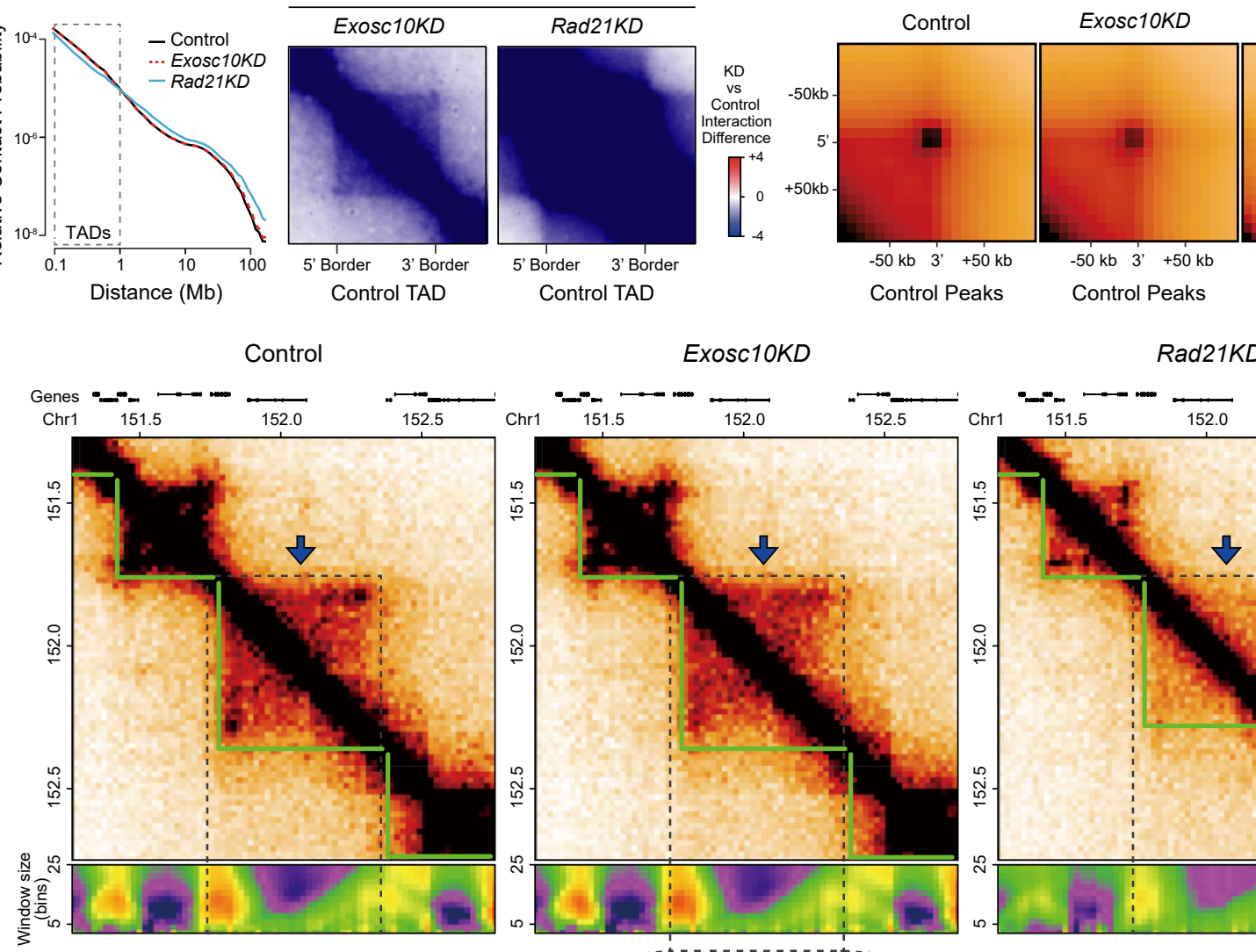

Rad21KD

Exosc10KD

Rad21KD
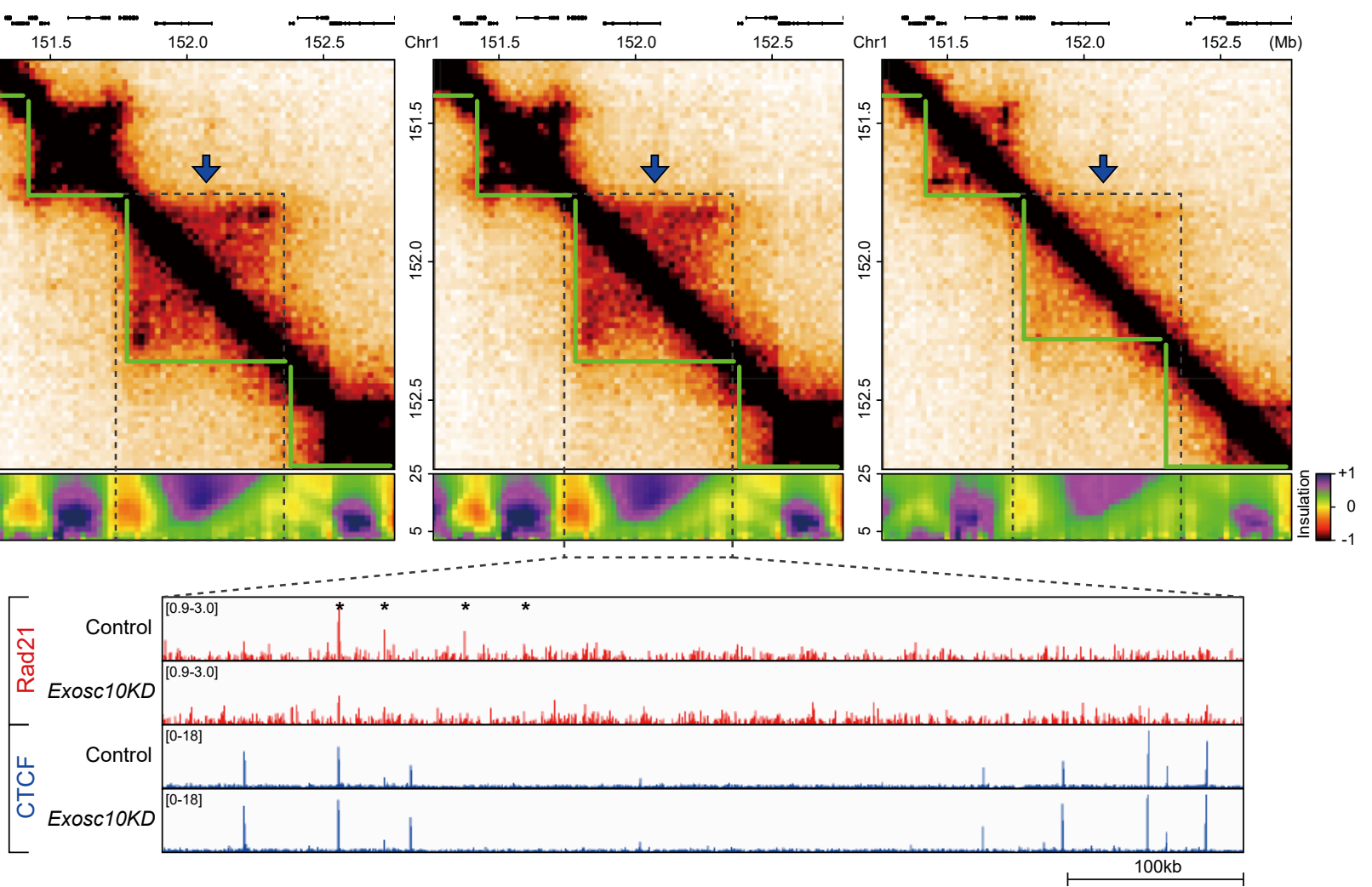
Figure 1 - figure supplement 1

A $\quad$ Rad21 peaks $(n=867)$

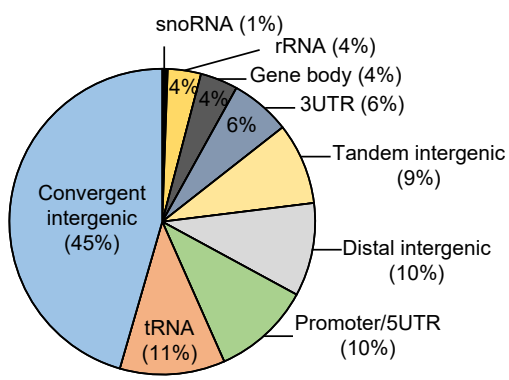

B

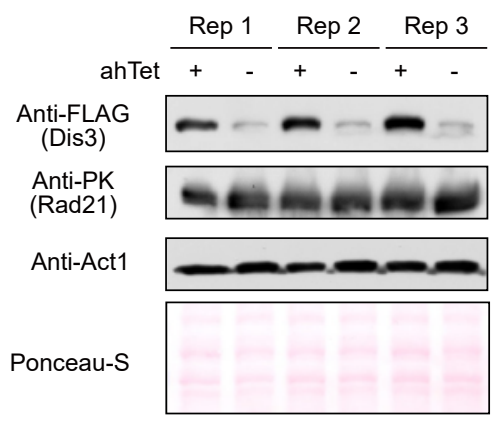

E
C

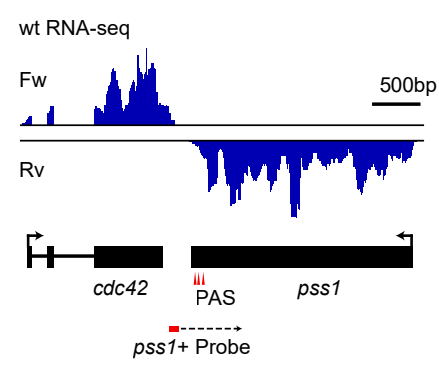

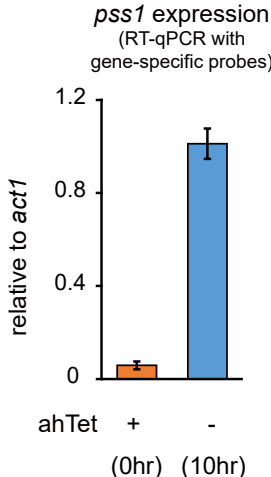

$\mathbf{F}$
D

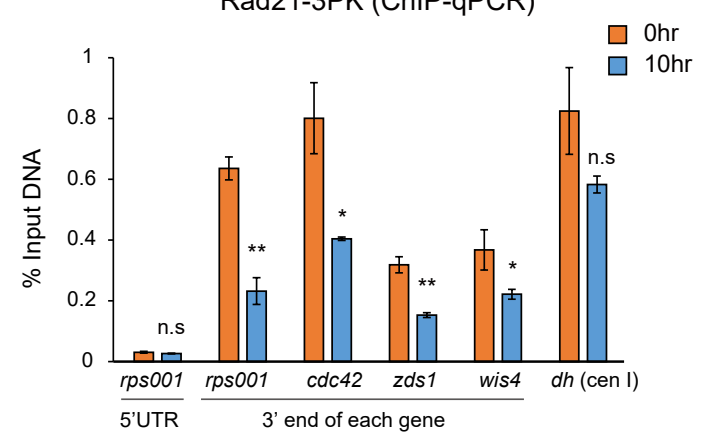

G

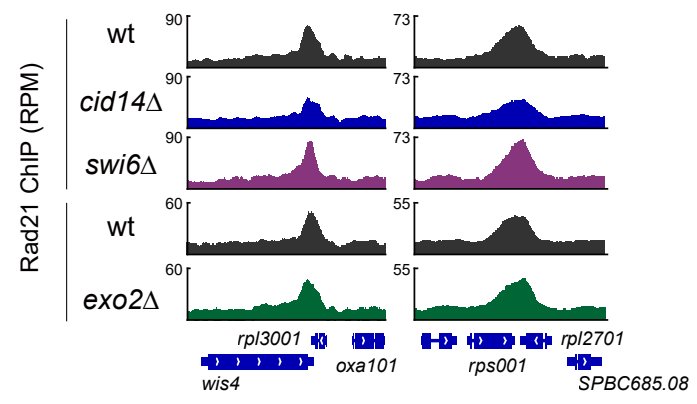

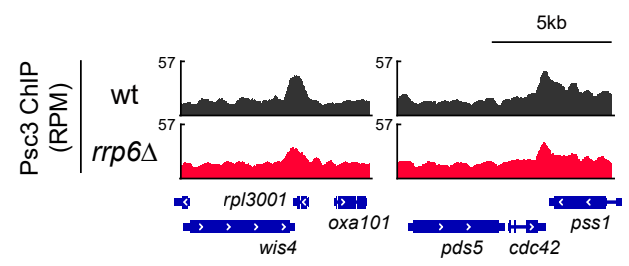

H

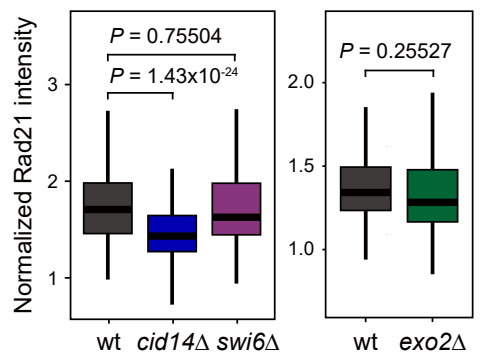

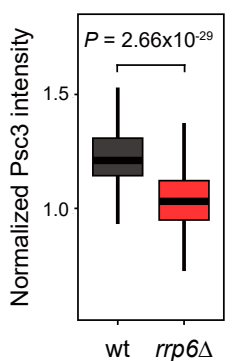

I

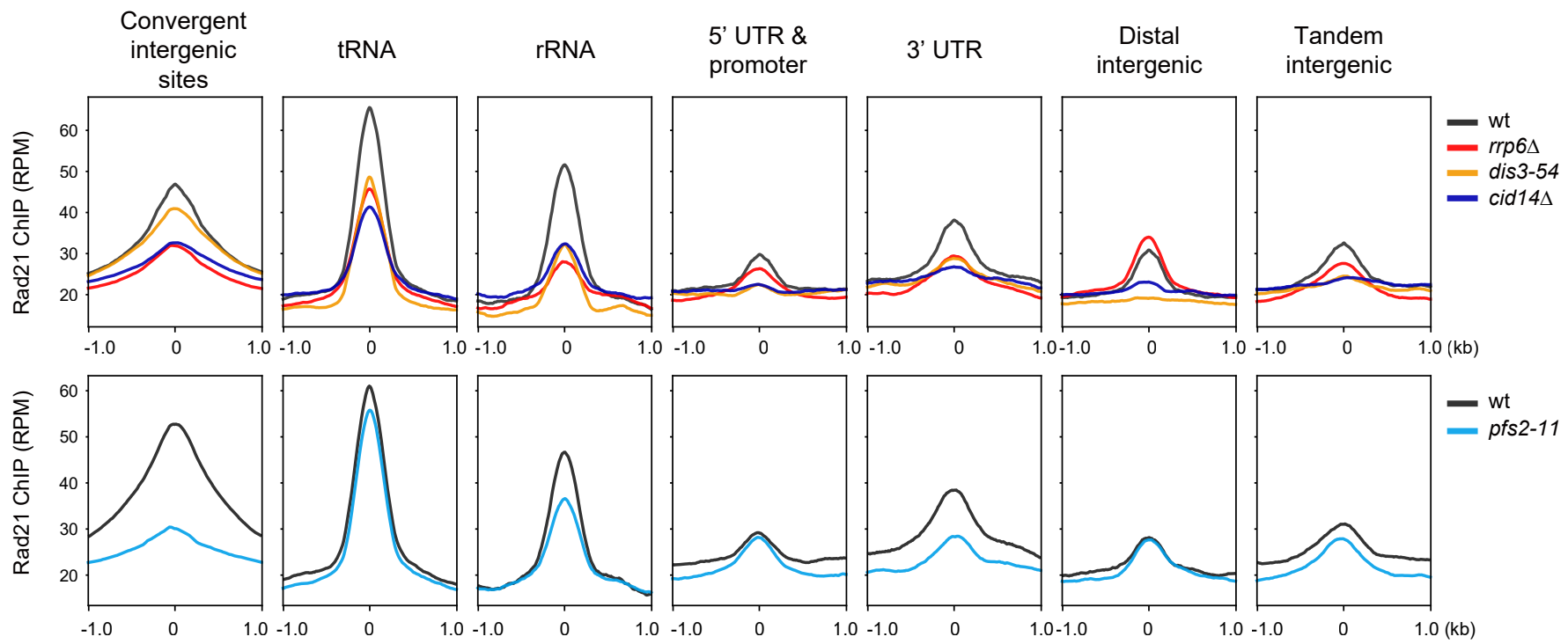




\section{Figure 1 - figure supplement 2}

A

centromere 1

(Chrl:3,745,000-3.800,000)

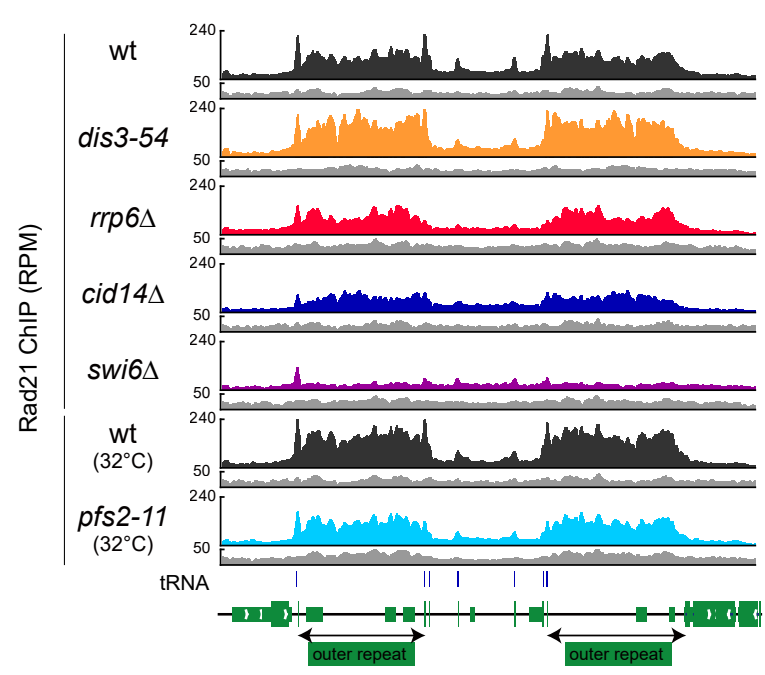

tellR

(Chrl:5,520,000-5,579,134)

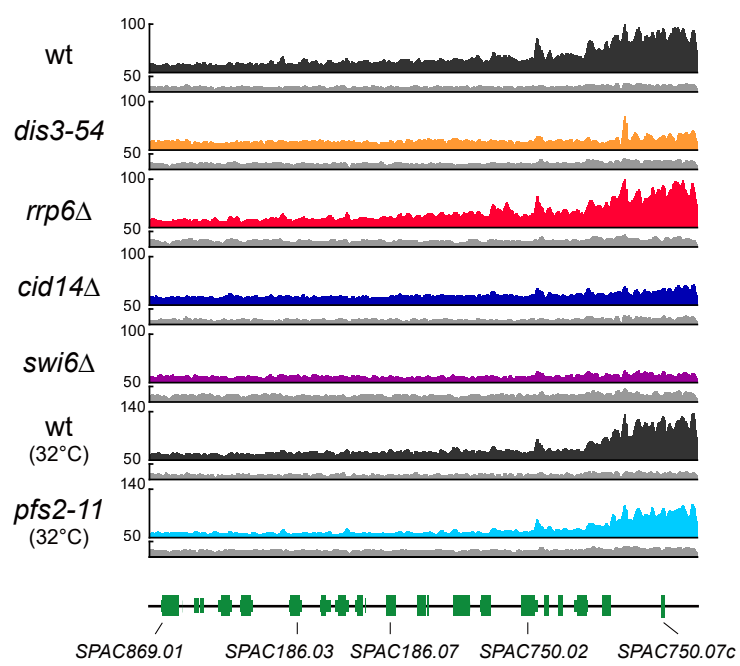

B

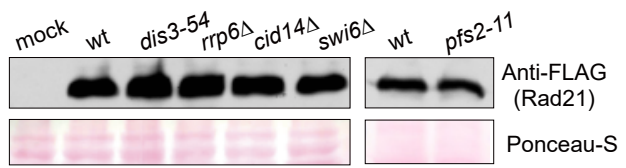


Figure 2 - figure supplement 1

A

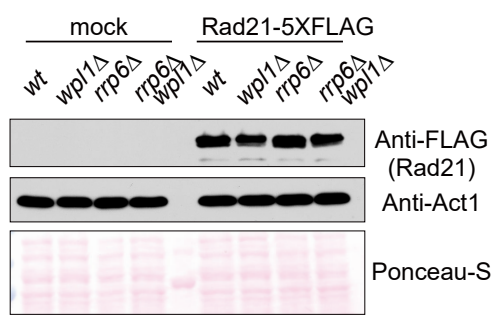

B

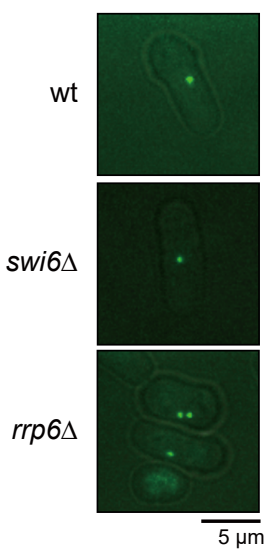

C

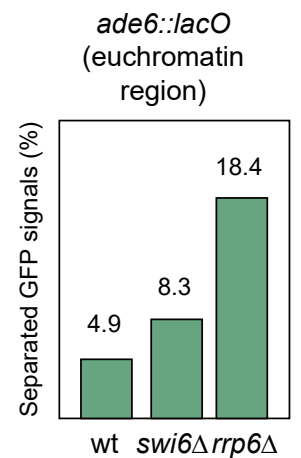

D

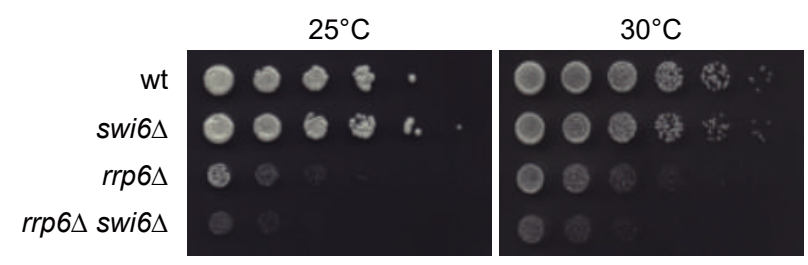


Figure 3 - figure supplement 1

A
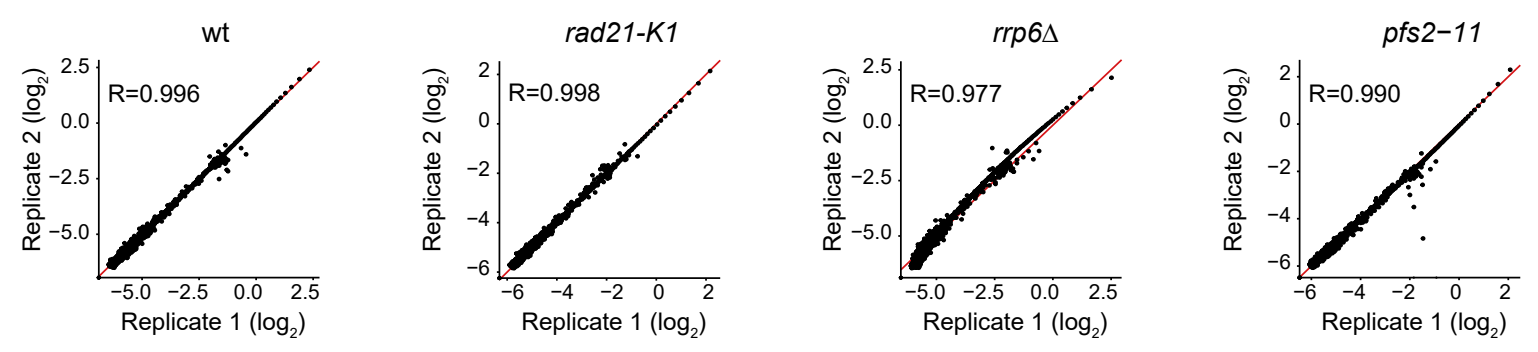

B

C

\begin{tabular}{|c|c|c|c|c|c|c|c|c|}
\hline 1.00 & 0.99 & 0.90 & 0.89 & 0.84 & 0.84 & 0.86 & 0.87 & rad21-k1 Rep 1 \\
\hline 0.99 & 1.00 & 0.90 & 0.89 & 0.84 & 0.84 & 0.86 & 0.87 & rad21-k1 Rep 2 \\
\hline 0.90 & 0.90 & 1.00 & 0.99 & 0.95 & 0.95 & 0.93 & 0.94 & pfs2-11 Rep 1 \\
\hline 0.89 & 0.89 & 0.99 & 1.00 & 0.94 & 0.94 & 0.93 & 0.94 & pfs2-11 Rep 2 \\
\hline 0.84 & 0.84 & 0.95 & 0.94 & 1.00 & 0.99 & 0.93 & 0.96 & wt Rep 1 \\
\hline 0.84 & 0.84 & 0.95 & 0.94 & 0.99 & 1.00 & 0.93 & 0.96 & wt $\operatorname{Rep} 2$ \\
\hline 0.86 & 0.86 & 0.93 & 0.93 & 0.93 & 0.93 & 1.00 & 0.97 & $r r p 6 \Delta \operatorname{Rep} 1$ \\
\hline 0.87 & 0.87 & 0.94 & 0.94 & 0.96 & 0.96 & 0.97 & 1.00 & $\operatorname{rrp} 6 \Delta \operatorname{Rep} 2$ \\
\hline 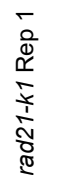 & 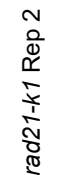 & 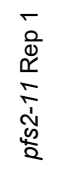 & 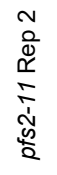 & $\begin{array}{l}\bar{c} \\
\stackrel{0}{0} \\
\alpha \\
\vdots \\
\vdots\end{array}$ & 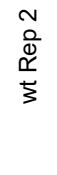 & $\begin{array}{l}\bar{c} \\
\stackrel{0}{0} \\
\alpha \\
\Delta \\
0 \\
\vdots\end{array}$ & $\begin{array}{l}\mathfrak{N} \\
o \\
Q \\
\alpha \\
4 \\
0 \\
2\end{array}$ & $\begin{array}{l}\text { Pairwise similarity } \\
\text { scores (SCC) } \\
\\
0.7\end{array}$ \\
\hline
\end{tabular}

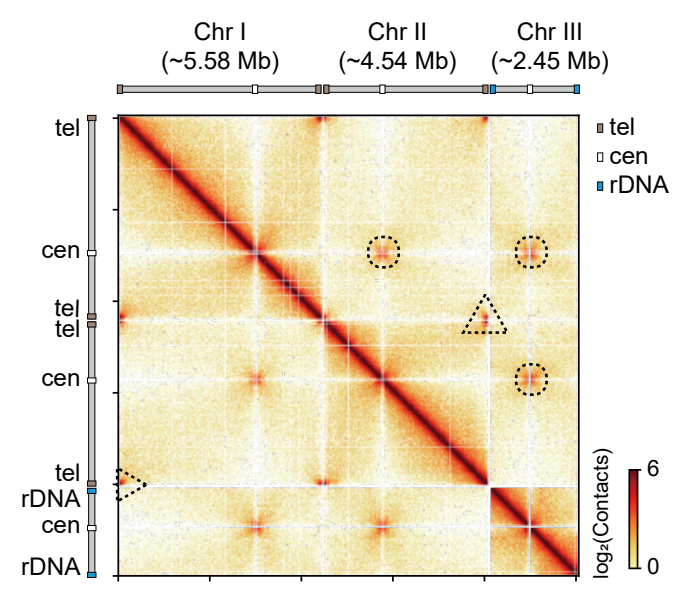


Figure 3 - figure supplement 2

A

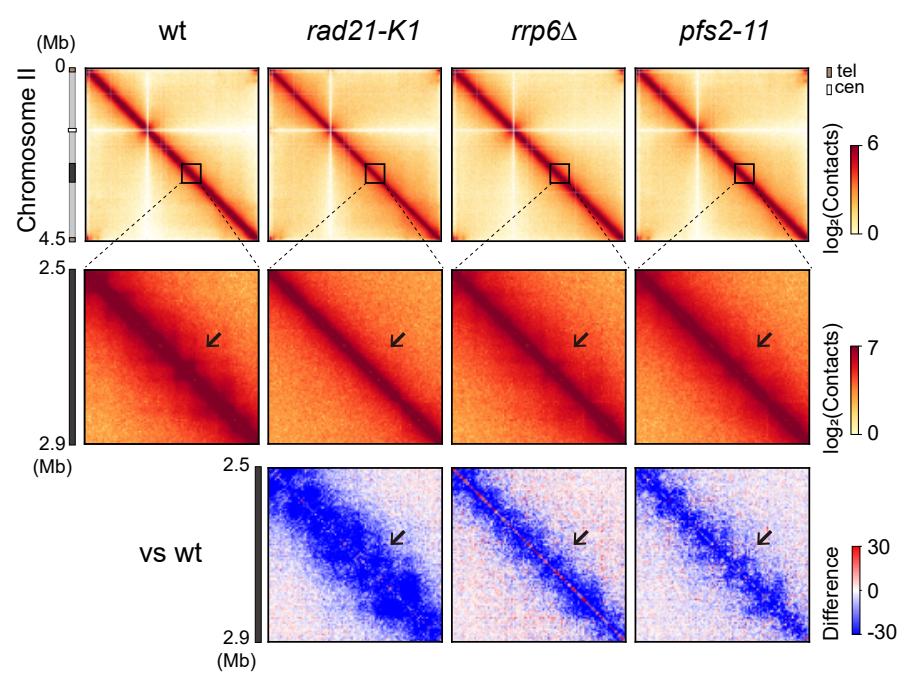

B

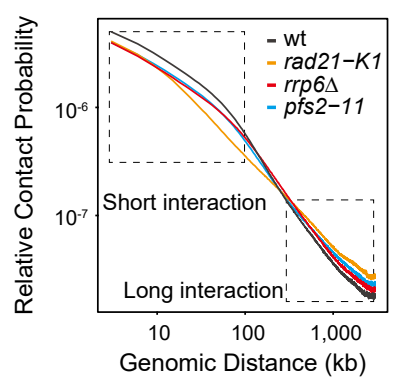

C

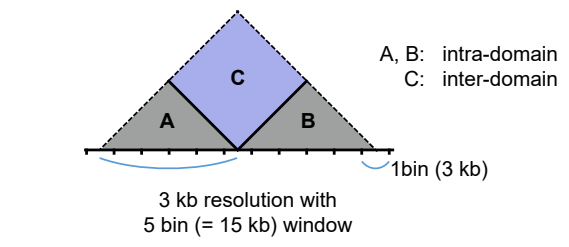

$D=\frac{A+B}{C} \quad$ Border Strength $=\frac{D-\text { mean }(D)}{S D(D)}$

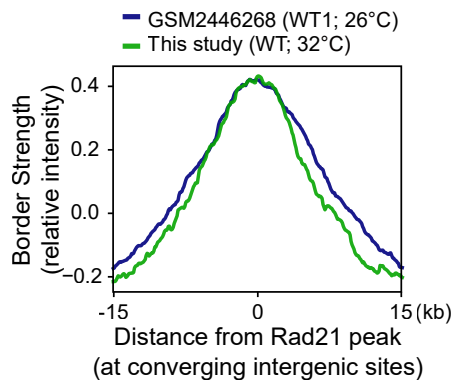

E

$\mathbf{F}$
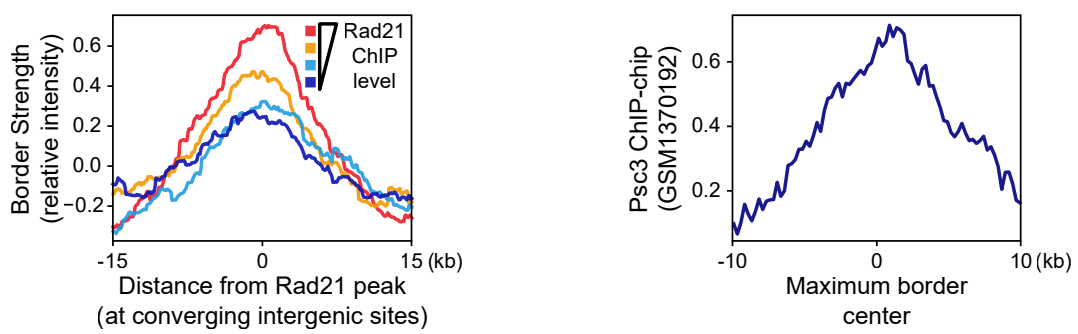

G

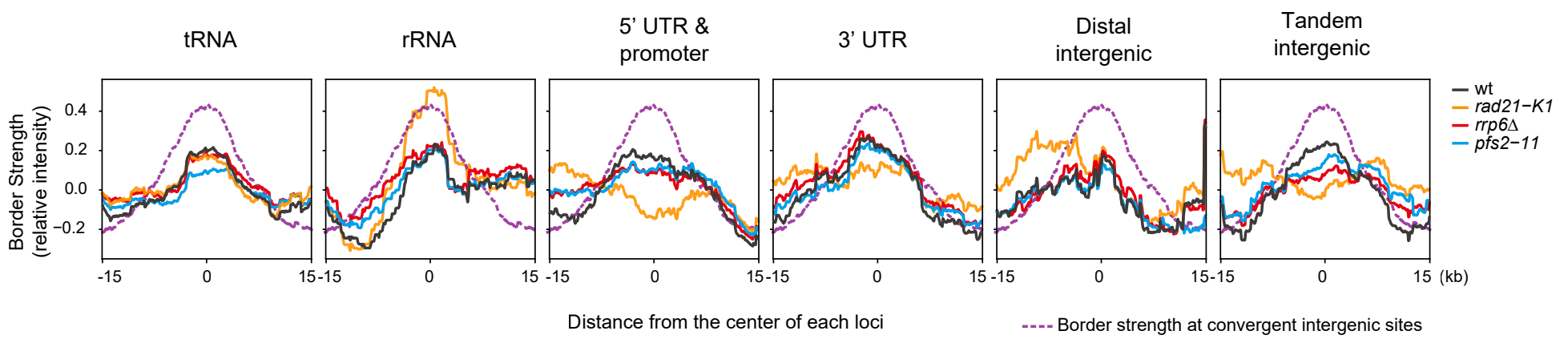


Figure 3 - figure supplement 3

A

dis3-54

(wild-type at $34^{\circ} \mathrm{C}$ )

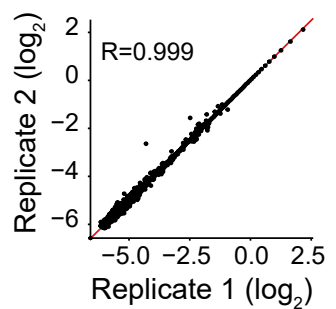

B

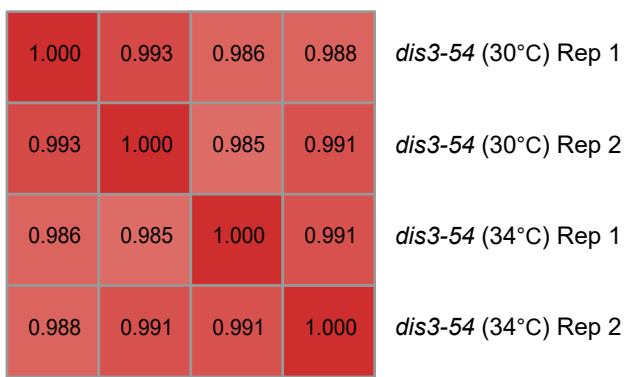

dis3-54 (defect at $30^{\circ} \mathrm{C}$ )

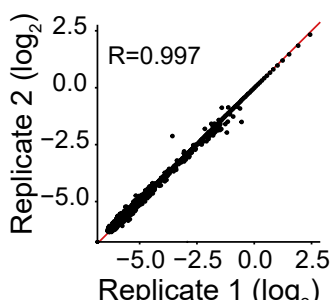

D

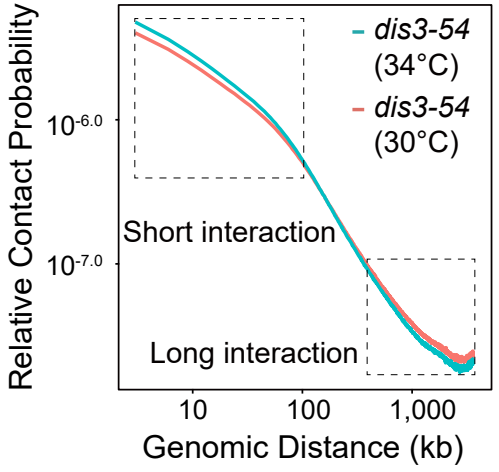

E
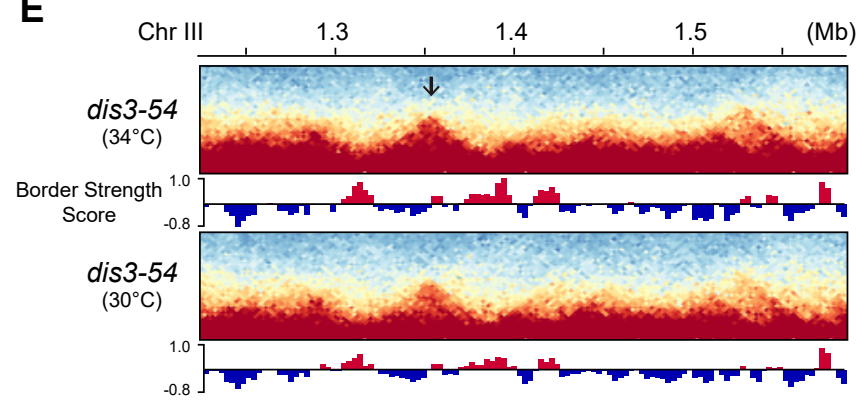

$\mathbf{F}$

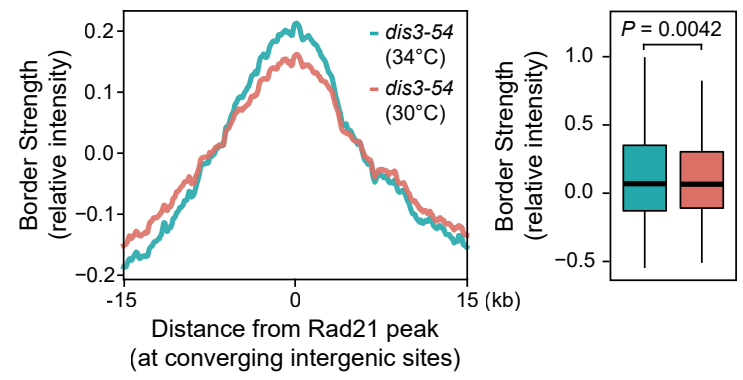

G

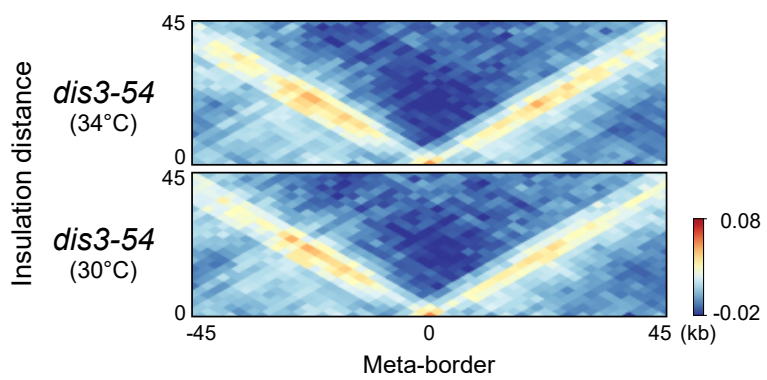




\section{Figure 4 - figure supplement 1}

A
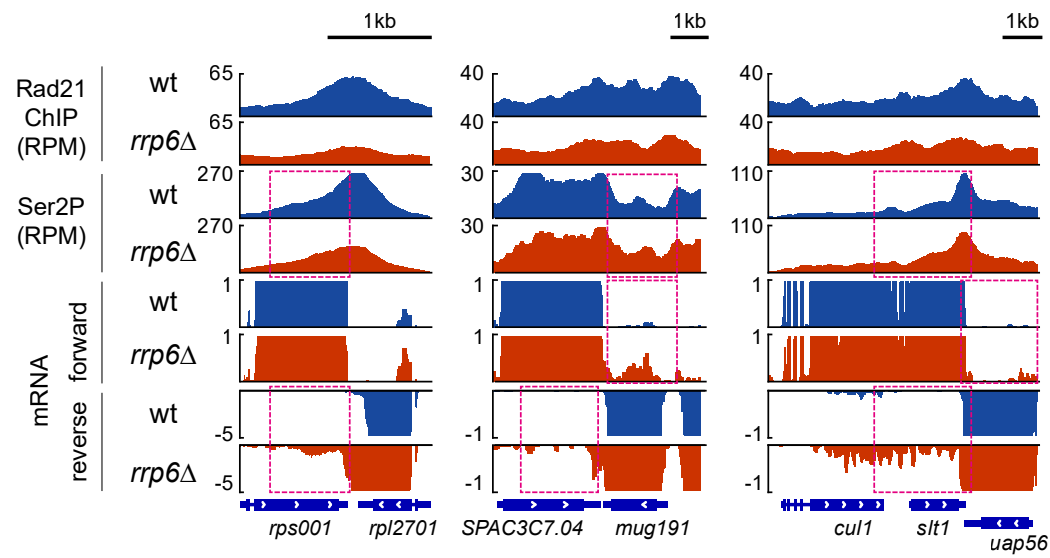

B

Rad21 peaks enriched at convergent intergenic regions $\rightarrow$ Grouped by Seb1 enrichment levels

C

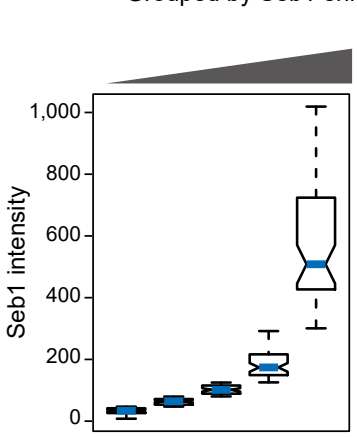

Seb1

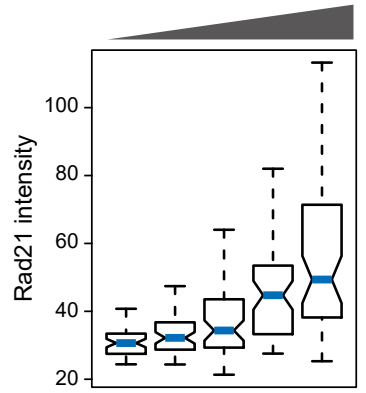

Seb1

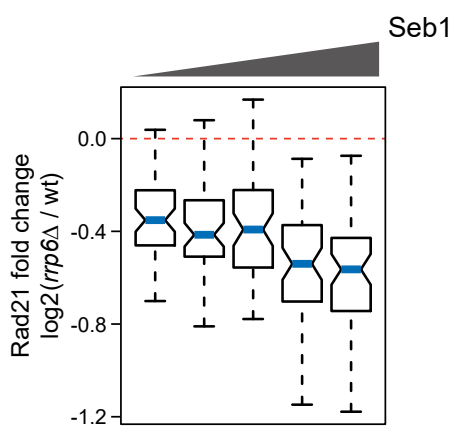

Rad21 peaks enriched at convergent intergenic regions $\rightarrow$ Grouped by Pcf11 enrichment levels

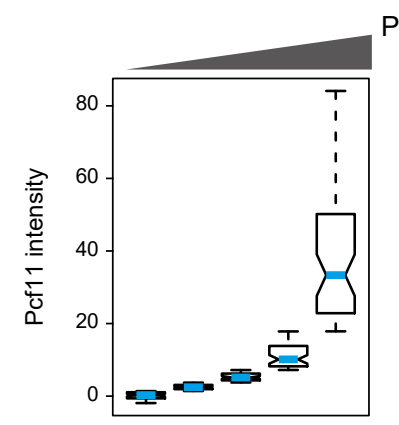

\section{Pcf11}

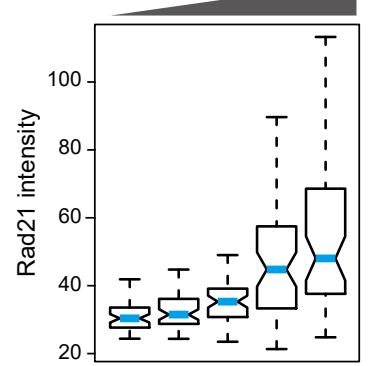

Pcf11

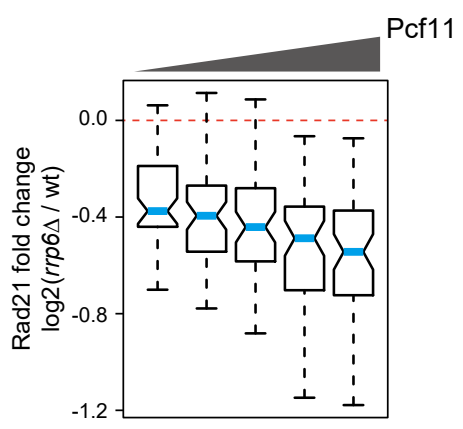

D

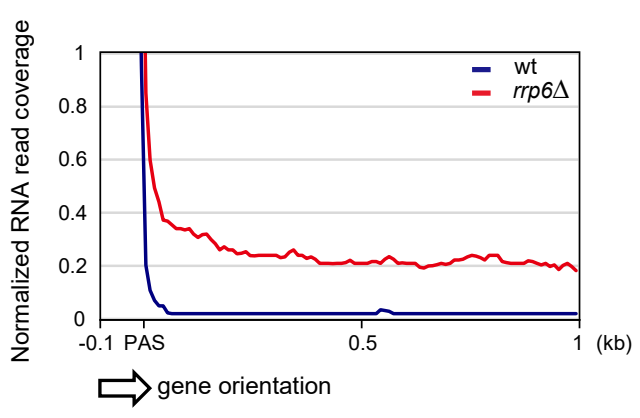

E

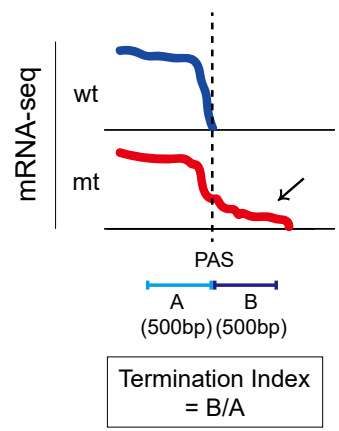

$\mathbf{F}$

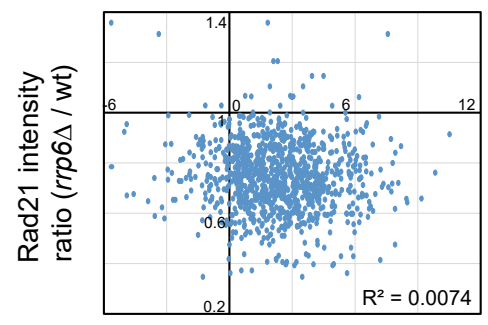

readthrough intensity (termination index) $\log _{2}(\operatorname{rrp} 6 \Delta / \mathrm{wt})$ 
Figure 5 - figure supplement 1

A

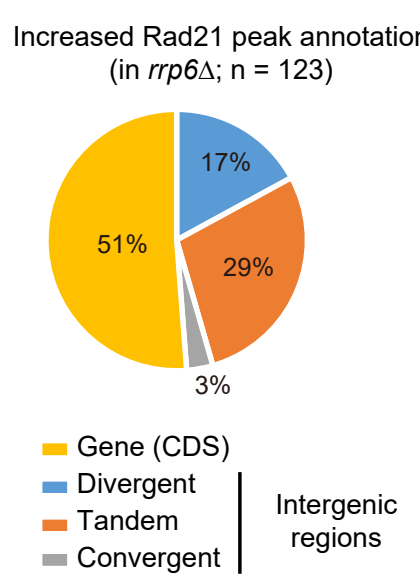

B

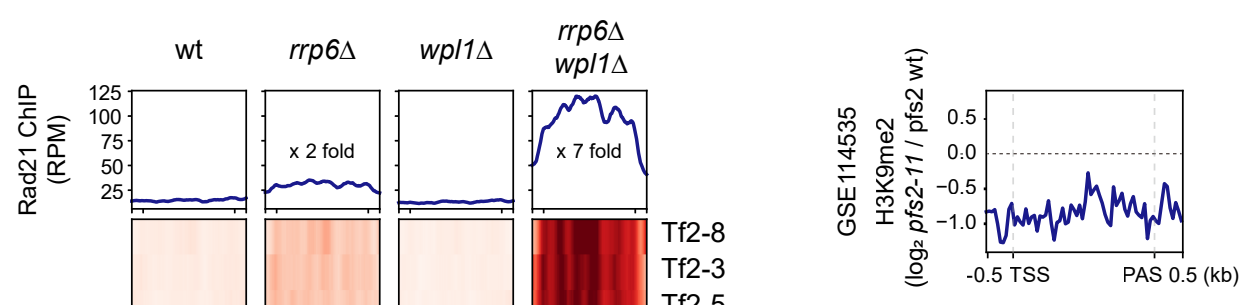

D

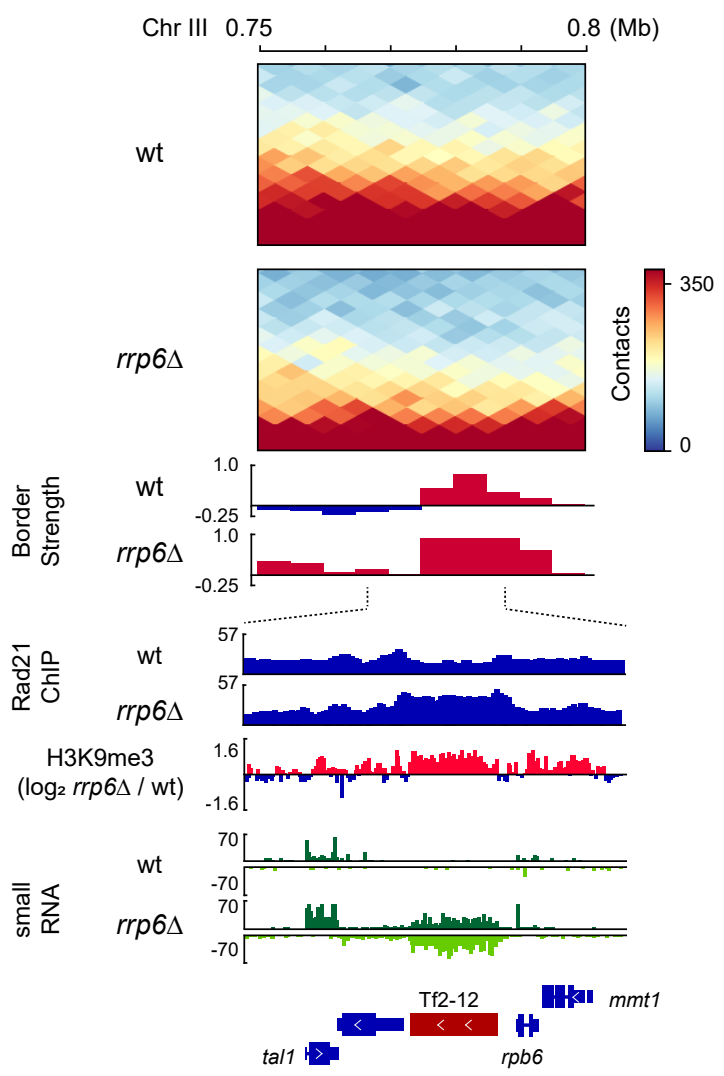


Figure 6 - figure supplement 1

A

B
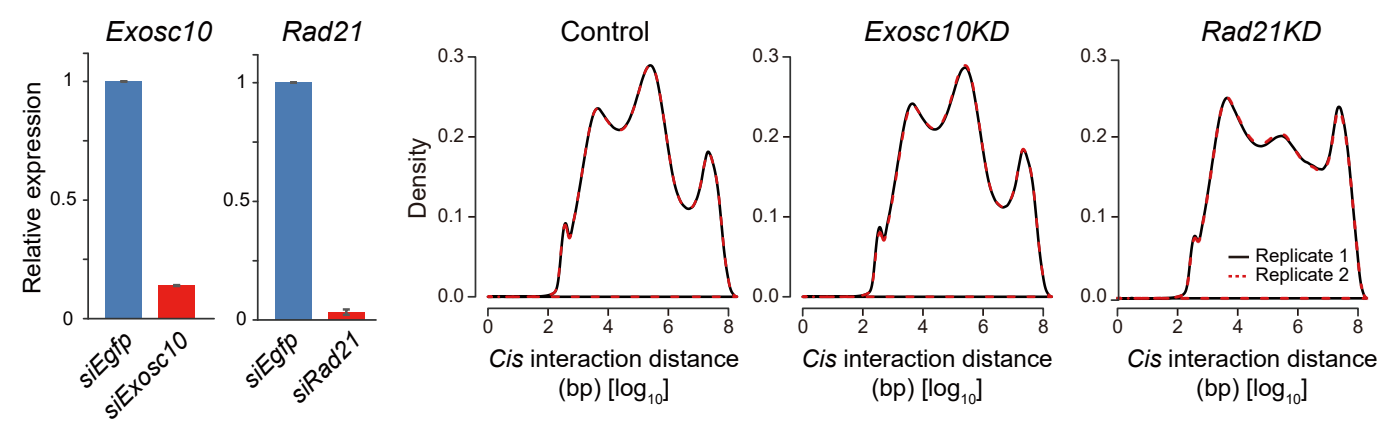

D

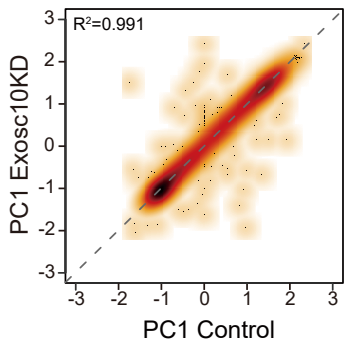

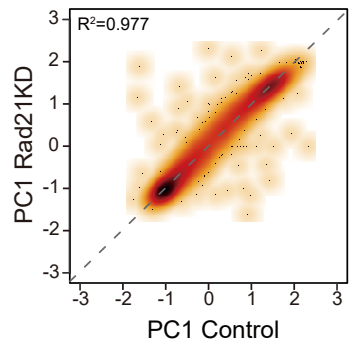

E

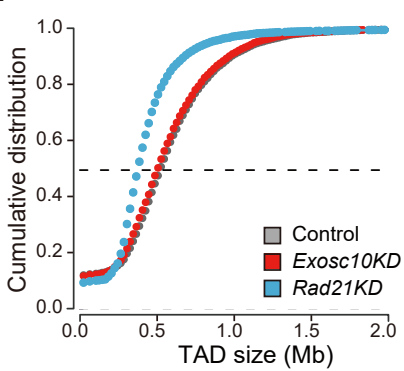

C

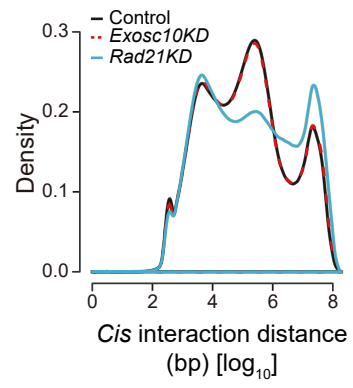

G
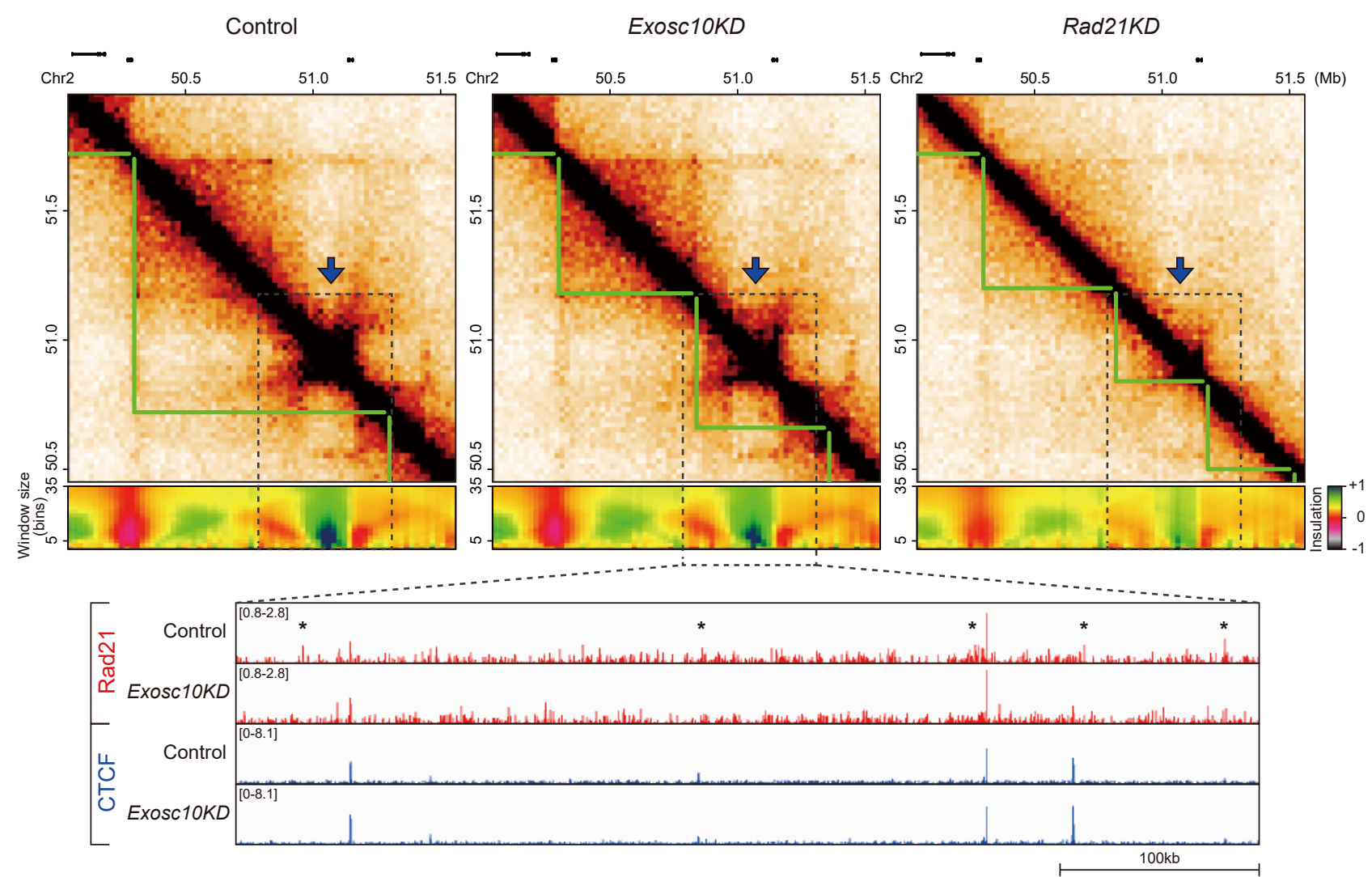